\title{
Empirical condition of betatron resonances with space charge
}

\author{
K. Kojima, H. Okamoto, ${ }^{*}$ and Y. Tokashiki \\ Graduate School of Advanced Sciences of Matter, Hiroshima University, \\ 1-3-1 Kagamiyama, Higashi-Hiroshima 739-8530, Japan
}

(Received 13 November 2018; published 25 July 2019)

\begin{abstract}
This paper addresses the generalization of the single-particle betatron resonance condition derived by Courant and Snyder more than a half century ago. A two-dimensional resonance condition including the effect of space-charge interaction was recently conjectured from one-dimensional Vlasov predictions made by Sacherer, Okamoto, and Yokoya [K. Ito et al., Phys. Rev. Accel. Beams 20, 064201 (2017)]. The condition is remarkably simple which only contains a few measurable quantities and indicates the possibility that twice as many resonance stop bands as expected from the conventional incoherent picture may exist at high beam density. Self-consistent multiparticle simulations are performed systematically to locate low-order stop bands in the tune diagram. The proposed betatron resonance formula is shown to explain the basic feature of numerical observations, which suggests that no serious incoherent resonance is activated inside the phase-space core of a dense beam matched to the external linear focusing potential. It is confirmed that the coherent tune-shift factor of any collective mode is less than unity and practically considered as a constant over the whole tune space in a typical high-intensity storage ring. The procedure for finding the optimum operating point of the ring is discussed on the basis of the coherent picture instead of the commonly used picture relying on the concept of incoherent tune spread. Despite years of theoretical efforts by many researchers, the coherent resonance concept is still not being employed for the construction of a stability tune diagram. We here provide a simple prescription to draw the diagram quickly. The present study also indicates the possibility of complete suppression of emittance exchange on particular difference resonances by choosing a proper emittance ratio.
\end{abstract}

DOI: 10.1103/PhysRevAccelBeams.22.074201

\section{INTRODUCTION}

Resonance is inevitable in modern particle accelerators composed of a periodic array of identical alternatinggradient (AG) beam focusing lattices. The machine operating point has to be put sufficiently away from dangerous low-order resonance lines along which serious emittance growth and resultant beam loss may occur. Although the linear (second-order) force is used to focus the beam, resonances of higher orders can take place in reality due to unavoidable error fields. Even if the external force is perfectly linear, the nonlinear nature of the Coulomb interaction excites a variety of nonlinear resonances at high beam density.

The classical single-particle resonance condition given by Courant and Snyder can be written as

\footnotetext{
*Corresponding author. okamoto@sci.hiroshima-u.ac.jp

Published by the American Physical Society under the terms of the Creative Commons Attribution 4.0 International license. Further distribution of this work must maintain attribution to the author(s) and the published article's title, journal citation, and DOI.
}

$$
k \nu_{0 x}+\ell \nu_{0 y}=n,
$$

where $\left(\nu_{0 x}, \nu_{0 y}\right)$ are the horizontal and vertical bare betatron tunes per lattice period or around the ring, and $(k, \ell, n)$ are integers [1]. The driving term of this resonance is proportional to $x^{|k|} y^{|\ell|}$ whose order is $|k|+|\ell|(\equiv m)$. Equation (1) is one of the most important formulas in beam dynamics and actually appears in most standard textbooks [2-5]. When either $k$ or $\ell$ is zero, we have

$$
m \nu_{0}=n,
$$

where $\nu_{0}$ denotes $\nu_{0 x}$ or $\nu_{0 y}$. Under the condition (2), the resonant instability develops in one of the two transverse degrees of freedom.

The recent trend toward higher beam density and/or power has made it indispensable to take the effect of spacecharge interaction correctly into account in the resonance analysis. The interparticle Coulomb force plays a crucial role even at low current when the beam is strongly compressed in phase space by a state-of-the-art cooling technique [6,7]. In any case, the natural repulsive force weakens the artificial focusing force from quadrupole magnets, leading to the reduction of effective betatron 
tunes down to $\nu_{x}\left(<\nu_{0 x}\right)$ and $\nu_{y}\left(<\nu_{0 y}\right)$ in both transverse directions. The magnitudes of the tune reduction, $\nu_{0 x}-\nu_{x}\left(\equiv \Delta \nu_{x}\right)$ and $\nu_{0 y}-\nu_{y}\left(\equiv \Delta \nu_{y}\right)$, are referred to as incoherent tune shifts. The so-called incoherent resonance condition is obtained by simply replacing the bare tunes in Eqs. (1) and (2) by the space-charge-depressed tunes, namely,

$$
k \nu_{x}+\ell \nu_{y}=n .
$$

For noncoupling instability limited to one of the two transverse degrees of freedom, we have

$$
m\left(\nu_{0}-\Delta \nu\right)=n,
$$

where $\Delta \nu$ represents either $\Delta \nu_{x}$ or $\Delta \nu_{y}$. This type of resonance should not be confused with the single-particle resonance that occurs under the condition (1) with no space-charge-induced tune shift.

The incoherent tune shifts $\left(\Delta \nu_{x}, \Delta \nu_{y}\right)$ cannot be determined uniquely. They may take different values depending on which particle we observe. The effective tunes $\left(\nu_{x}, \nu_{y}\right)$ of the particles forming a particular beam cover a finite area in the tune diagram, which is called the incoherent tune spread. As schematically illustrated in Fig. 1, the conventional incoherent concept requires the designer to choose the machine operating point $\mathrm{P}$ in the tune diagram such that the tune-spread area does not cross nearby loworder single-particle resonance lines predicted by Eq. (1).

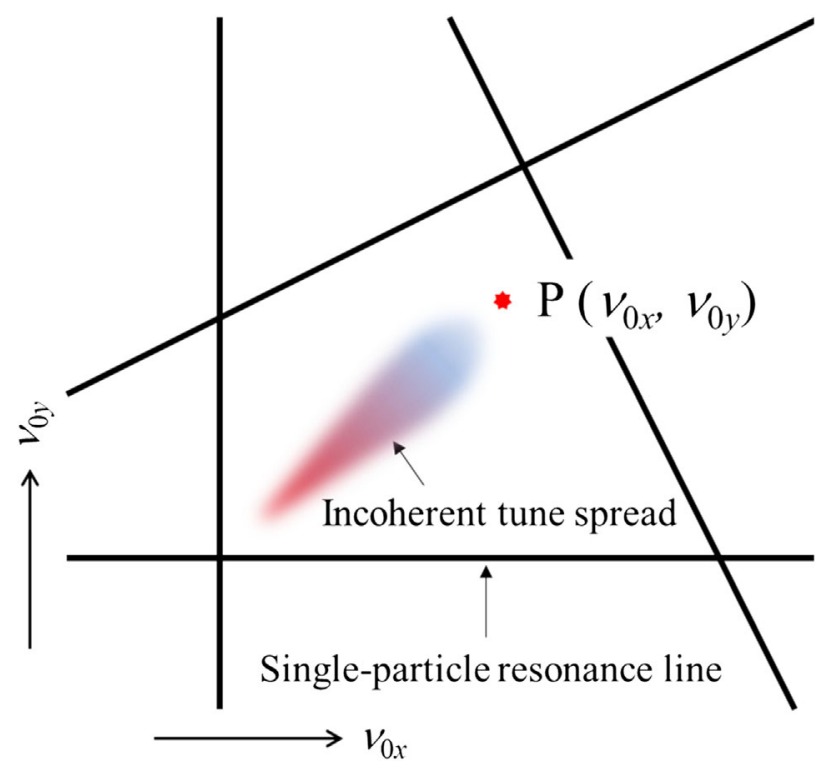

FIG. 1. Determination of a machine operating point on the basis of the conventional incoherent concept. The operating bare tunes $\left(\nu_{0 x}, \nu_{0 y}\right)$ of a machine are chosen so that the incoherent tune spread (shaded area) does not cross low-order single-particle resonance lines. A certain plausible distribution function has to be assumed to estimate the tune-spread area. See Appendix A for an example of the tune spread in the Gaussian distribution.
This kind of stability chart has been often employed in the community to explain space-charge-induced beam loss in a high-intensity hadron machine or to decide the optimum operating point [8], but we will show this picture to be naive at best.

The incoherent tune spread depends on what particle distribution the beam possesses in phase space. It becomes different for different types of distribution functions even if the beam current and average density are kept unchanged. As an example, consider the Kapchinskij-Vladimirskij $(\mathrm{KV})$ distribution that has the uniform particle density in real space [9]. Since the resultant space-charge potential is quadratic, no tune spread exists in the KV beam. Any choice of the operating point would then be permitted as long as we follow the common rule based on the incoherent concept. It is, however, well-known that even a completely uniform distribution as the $\mathrm{KV}$ type does have many instability bands of finite widths [10-12]. Besides, the $\mathrm{KV}$ beam is intrinsically unstable against perturbation at high density [11]. This indicates the crucial importance of self-consistency in the stability analysis of space-chargedominated beams.

The incoherent resonance condition can be derived from the frozen space-charge model (FSM) that assumes a rigid beam core. The particle distribution of the whole beam is never affected in the FSM even when the motion of the reference particle loses its stability. This is nothing but a simplifying assumption that allows a quick estimate of the space-charge effect on intense beam resonances. Even though the FSM may be a convenient means for judging a possible operating condition of any machine, we should keep it in mind that the model was originally introduced because of the difficulty of treating the collective beam behavior in a self-consistent manner. Considering the reachable distance of the Coulomb interaction, the betatron motions of individual particles in a dense beam core cannot be independent but surely have some correlation. The instability of the beam's main body is expected to develop collectively.

Note that the beam core must be defined in full phase space rather than in real space $[13,14]$. It is generally impossible to carry out accurate separation of halo (tail) particles from the core simply by looking at the beam profile in real space. At any moment, many halo particles are hidden behind the real-space core of the beam.

One of the most trustable approaches to space-charge issues is the use of the Vlasov-Poisson equations $[15,16]$. The Vlasov formalism is self-consistent and includes all relevant physical processes except for interparticle Coulomb collisions whose effect is negligible in the present study $[17,18]$. It is thus definitely superior to the FSM. Much effort has been devoted by many researchers to establishing the concept of coherent resonances with selfconsistent techniques [10-12,19-29]; see an excellent review by Baartman in Ref. [30]. The first pioneering 
work was done by Sacherer who mathematically solved the Vlasov-Poisson equations to explore the stability of an intense one-dimensional (1D) beam [20]. Under the smooth approximation, he derived the resonance condition

$$
m\left(\nu_{0}-C_{m h} \Delta \nu\right)=n,
$$

where $C_{m h}$ is a constant factor with two indices representing the azimuthal $(m)$ and radial $(h)$ mode numbers. As Sacherer assumed a spatially uniform beam, all particles have an identical tune shift $\Delta \nu$, thus no tune spread. Baartman pointed out that the modes with $m \neq h$ are invisible in simulations and probably unimportant in practice. $C_{m h}$ in Eq. (5) may, therefore, be replaced simply by $C_{m}$. Theoretically, $C_{m}<1$ for all $m$ numbers.

Strictly speaking, the coherent estimate should not directly be compared with the incoherent estimate. The Vlasov equation treats the distribution function of the whole beam in phase space rather than the trajectories of individual particles. Since the beam is regarded as a sort of continuum, there is no room for the incoherent motion to come in. The left-hand side of Eq. (5) corresponds not to the incoherent tune but to the tune of the collective oscillation mode of the $m$ th order.

The Sacherer's analytic theory was extended by Gluckstern to a coasting round beam, i.e., the case where $\nu_{0 x}=\nu_{0 y}$ [11]. Hofmann et al. later proposed a twodimensional (2D) coherent resonance condition, adding a correction term to Eq. (3):

$$
k \nu_{x}+\ell \nu_{y}+\Delta \omega=n,
$$

where $\Delta \omega$ is the coherent tune shift away from the incoherent resonance condition [29]. The incoherent tunes in Eq. (6) have been defined assuming the uniform particle density. The coherent shift $\Delta \omega$ is a complicated function of several parameters including the integers $(k, \ell)$, the emittance ratio, etc. A few different values of the coherent tuneshift factor are theoretically possible in the $2 \mathrm{D}$ case even for a specific order of mode with $m=h$ [25,30]. If all of them are practically important, stop-band splitting may be observed in self-consistent numerical simulations and experiments. Additional indices are then certainly needed for the factor $C_{m}$ to distinguish these instabilities of the same order $m$. The numerical results in the following sections, however, show no such splitting effect clearly.

We here concentrate on the transverse betatron dynamics, ignoring the synchrotron motion. The present discussion is restricted basically to coasting beams. In Sec. II, we introduce the 2D coherent resonance condition recently conjectured in Ref. [31]. We then proceed to systematic particle-in-cell (PIC) simulations. A simple linear focusing force is considered first in Sec. III to figure out the distribution of resonance stop bands induced solely by the space-charge potential. Several different matching conditions are taken initially to check if the proposed coherent resonance formula can explain the parameterdependence of self-consistent numerical results. An interesting finding on difference resonance is also given in this section. External perturbing forces are switched on in Sec. IV, which enables us to make a better estimate of the coherent tune-shift factor. Three types of initial distribution functions, i.e., Gaussian, waterbag, and parabolic, are used for the estimate. It is confirmed that the tune-shift factor is smaller than unity for any collective oscillation modes. In Sec. V, we apply the coherent formula to the lattice of an existing high-intensity ring. Finally, concluding remarks are made in Sec. VI.

\section{EMPIRICAL 2D COHERENT RESONANCE CONDITION}

The periodic nature of the external driving force is essential to resonance excitation. No resonance takes place under uniform external fields even at very high beam density. Sacherer employed the smooth approximation in his Vlasov theory, so all collective modes were stable. Some periodic perturbation, therefore, has to be introduced eventually in order to conclude the resonance condition as in Eq. (5). Such a mathematical procedure corresponds to adding weak imperfections to the ideal lattice. Okamoto and Yokoya (OY) generalized Sacherer's approach, solving the 1D Vlasov-Poisson equations without the smooth approximation [27]. By the help of the waterbag model, they derived a purely analytic description of coherent resonance that can be applied to arbitrary lattice structures. The OY theory says that the beam becomes unstable when two azimuthal modes $m_{1}$ and $m_{2}$ couple. These mode numbers are not necessarily equal but need to satisfy $m_{1}+$ $m_{2}=$ even and $m_{1} m_{2}<0$ for resonance excitation. The instability is particularly severe when $\left|m_{1}\right|=\left|m_{2}\right|=m$. The general resonance formula can then be simplified to

$$
m\left(\nu_{0}-C_{m} \Delta \bar{\nu}\right)=\frac{n^{\prime}}{2}
$$

that includes Eq. (5) as a special case. Here, $n^{\prime}$ is an integer, and $\Delta \bar{\nu}$ represents the root-mean-squared (rms) tune shift that can be related to the rms tune depression $\eta$ as $\Delta \bar{\nu}=(1-\eta) \nu_{0} . \Delta \bar{\nu}$ is much less than the maximum incoherent tune shift of a Gaussian beam as demonstrated in Appendix A. Equation (7) has been linearized with respect to the rms tune shift for the sake of simplicity, assuming relatively low beam density. The instability of a smaller $m$ is stronger in the absence of external driving fields [27]. The linear-mode $(m=2)$ resonance corresponds to the so-called "envelope instability" explored previously by many researchers $[9,10,12,21,22,32]$.

No artificial driving perturbation is necessary to draw the condition (7) from the Vlasov equation. Despite the perfect linearity of the external potential, all kinds of nonlinear 
modes turn out to be excited by the nonlinear nature of the Coulomb self-field potential. Of practical importance is the factor $1 / 2$ on the right-hand side of Eq. (7), which is missing in Eq. (5) and other conventional resonance formulas. This extra factor suggests the possibility that there may exist twice as many resonance stop bands as predicted by common resonance theories. The resonances for $n^{\prime}=2 n$ are enhanced by artificial imperfection fields and nonlinear magnets, no matter whether the beam density is high or low. In contrast, the resonances for odd $n^{\prime}$ are weakened at lower beam intensity and eventually disappear at zero current limit.

The coherent resonance condition in Eq. (7) is a consequence resulting from the eigenvalue problem of the form $\boldsymbol{M} \cdot \boldsymbol{u}=\mu \boldsymbol{u}$ [27]. $\boldsymbol{M}$ is a real matrix depending on fundamental parameters such as the betatron tune and beam density. The eigenvalue can be expressed as $\mu=$ $\exp \left(-2 \pi i \Omega_{m}\right)$ with $\Omega_{m}$ being the tune of a coherent oscillation. The collective mode is stable provided $|\mu|=1$. The stability condition $[\operatorname{Re}(\mu)]^{2}+[\operatorname{Im}(\mu)]^{2}=1$ will eventually be broken if we keep increasing the beam density or move the operating point toward a resonance band. When the threshold is reached, we have $\operatorname{Re}(\mu)= \pm 1$ and $\operatorname{Im}(\mu)=0$, which means that $\Omega_{m}$ is either an integer or a half integer. This is a mathematical reason for the appearance of the parametric factor.

It is theoretically expected that the coherent oscillation of $m$ th order has two branches of tunes approximately given by $k_{1}+\Omega_{m}$ and $k_{2}-\Omega_{m}$ where $k_{1}$ and $k_{2}$ are integers [27]. The existence of these frequency components has been confirmed experimentally for the dipole $(m=1)$ and quadrupole $(m=2)$ modes $[33,34]$. The stability of the $m$ th-order mode will be affected when the two components merge, namely, $k_{1}+\Omega_{m}=k_{2}-\Omega_{m}$ that gives $\Omega_{m}=$ $\left(k_{2}-k_{1}\right) / 2$. We again conclude the necessity of the factor $1 / 2$ on the right-hand side.

We have emphasized the importance of the parametric resonance condition (7) since its discovery [27,31,35-41], presenting not only systematic numerical simulation data but also various experimental observations from the novel tabletop apparatus "S-POD" (Simulator of Particle Orbit Dynamics) at Hiroshima University [42-44]. S-POD reproduces, within a very compact ion trap [45], the collective behavior of an intense beam propagating through a wide range of AG lattice configurations; see Appendix B for some information about S-POD. The resonance formula in Eq. (7) had, however, attracted only little attention until recently. As a result, the common interpretations of severe resonances that occur at high intensity sometimes conflicted with ours. There still seem to be substantial differences in the understanding of nonlinear resonances [46].

The extension of the 1D coherent formula to 2D beams without the smooth approximation is of huge practical importance. Such an attempt was once made by Hofmann et al. for linear symmetric FODO and solenoidal channels
[12], but no 2D resonance formula beyond Eq. (6) had been given explicitly. Since it is hopeless to solve the 2D VlasovPoisson equations mathematically for arbitrary AG lattices, a plausible conjecture was made recently by the Hiroshima group [31]. The proposed 2D resonance condition has a remarkably simple form, in spite of the complex collective process behind:

$$
k\left(\nu_{0 x}-C_{m} \Delta \bar{\nu}_{x}\right)+\ell\left(\nu_{0 y}-C_{m} \Delta \bar{\nu}_{y}\right)=\frac{n^{\prime}}{2},
$$

where the horizontal and vertical rms tune shifts can be evaluated from $\Delta \bar{\nu}_{x(y)}=\left(1-\eta_{x(y)}\right) \nu_{0 x(0 y)}$ with $\eta_{x(y)}$ being the rms tune depressions. The linearization with respect to $\Delta \bar{\nu}_{x(y)}$ has again been done in Eq. (8), which should be no problem in the beam-intensity range of a typical storage ring. Equation (8) is reduced exactly to Eq. (7) in the case of purely horizontal or vertical resonance where $(k, \ell)=$ $(m, 0)$ or $(0, m)$. At zero current limit $\left(\eta_{x(y)} \rightarrow 1\right)$, it becomes identical to the single-particle resonance condition in Eq. (1) when $n^{\prime}=$ even. This formula has a few significant features compared with conventional resonance conditions:

(i) There is the factor $1 / 2$ on the right-hand side, unlike other well-known resonance formulas in Eqs. (1)-(6). This extra factor is of essential importance in practice because it leads to a two-fold increase of the density of resonances in the tune diagram.

(ii) The tune-shift factor $C_{m}$ depends only on the resonance order $m(=|k|+|\ell|)$. The coherent tune shift discussed in previous works are much more complex; it has a few indices and is even a function of such parameters as the beam ellipticity and the degree of the tune split, in other words, it depends on machine operating conditions $[25,29,30,47]$. Here, $C_{m}$ is simply a constant.

(iii) No model-dependent unobservables are included. The space-charge-induced tune shifts have not been treated very carefully in previous coherent formulas probably because most Vlasov theories rely on the KV model where no incoherent tune spread exists. Equation (8) is free from any incoherent quantities that cannot uniquely be defined for realistic nonuniform beams.

The generalization of the $1 \mathrm{D}$ condition (7) to $2 \mathrm{D}$ is not a trivial issue at all. The resonance conjecture in Eq. (8) was first reached in Ref. [31] through careful theoretical considerations and systematic S-POD experiments $[48,49]$. Hofmann added the parametric factor $1 / 2$ to the right-hand side of Eq. (6) in his recent monograph [47], which results in a resonance condition analogous to Eq. (8). The abovelisted points, however, still remain to be verified. This is a primary motivation of the present work.

The integer $n^{\prime}$ on the right-hand side of Eq. (8) is replaced by $N_{\mathrm{sp}} n^{\prime}$ for an ideal storage ring composed of $N_{\mathrm{sp}}$ lattice superperiods. The importance of the other driving 
harmonics depends on the strengths of random imperfection fields. Obviously, it is preferable to maintain the lattice periodicity around the ring as high as possible. Any new electromagnetic element that yields a non-negligible transverse potential had better be inserted every superperiod, if possible, to avoid breaking the original lattice symmetry.

External potential sources such as nonlinear errors and correction magnets can enhance the resonances of the corresponding orders only for even $n^{\prime}$ [27,31]. In the following, we call this type of resonance "external-fielddriven". The coherent resonance originating purely from the space-charge potential is referred to as "self-fielddriven" that occurs regardless of the parity of the driving harmonic number $n^{\prime}$. The strength of any resonance with even $n^{\prime}$ thus depends both on the beam density in phase space and on the degree of artificial imperfections in the machine lattice. At low beam density, all resonances with odd $n^{\prime}$ become negligible because their source is the spacecharge potential only. The growth rates of the self-fielddriven nonlinear resonances are generally low in a circular machine where the acceptable range of tune depression is limited. This type of collective resonance will, however, cause a non-negligible effect when an intense hadron beam stays in a ring for a long period at relatively low energy [31].

The resonance formula in Eq. (8) includes just a few physical parameters, all of which are measurable in principle. The bare tunes $\left(\nu_{0 x}, \nu_{0 y}\right)$ are determined once the machine operating point is chosen. Unlike the incoherent tune shifts $\left(\Delta \nu_{x}, \Delta \nu_{y}\right)$, the rms tune shifts $\left(\Delta \bar{\nu}_{x}, \Delta \bar{\nu}_{y}\right)$ or, in other words, the rms tune depressions $\left(\eta_{x}, \eta_{y}\right)$ can readily be calculated from the rms envelope equations as outlined in Appendix A. Since the envelope equations are insensitive to the phase-space distribution of particles [50], we can uniquely define these rms quantities whenever the beam perveance and emittances are known. The combinations of three integers $\left(k, \ell, n^{\prime}\right)$ that demand particular attention in practice are associated with the lattice design, the distribution and strengths of possible nonlinear external fields, etc. The only natural parameter artificially uncontrollable is the tune-shift constant $C_{m}$. If the coherent response to the AG lattice dominates the beam core after any resonance process is initiated, we shall realize that the $C_{m}$-factor below unity is indispensable to explain selfconsistent simulation data.

To avoid confusion, we stress the point that the purpose of regular resonance analysis is to clarify the stability of a matched beam core, i.e., the main body of a beam that has a particular stationary distribution of particles in phase space. The stability of a strongly mismatched beam, the dynamics of a nonstationary tail surrounding the core, and so forth, are different issues and thus of no primary interest to us here. The behavior of tail particles can approximately be studied, for example, with a sort of FSM called "particlecore model" (PCM). PCM is not intended for explaining the core resonance mechanism but aims at giving a rough picture of halo formation outside the core. This model has been used typically to estimate the maximum halo extent as a function of initial beam-size mismatch. Taking the Debye screening effect and mathematical simplicity into account, the most standard PCM assumes a uniform beam core executing the mismatch-induced breathing or quadrupole oscillation [51-53]. On the other hand, Eqs. (5) and (7) are obtained by solving the 1D Vlasov-Poisson equations in a perturbative manner. We first define a distribution function matched to the assumed lattice and then apply a small disturbance to see how it evolves in time. The disturbance grows under the coherent resonance conditions, instead of oscillating about the stationary state. The collective motion of a beam far from the stationary state or already collapsed due to some instability is beyond the scope of perturbative resonance theories.

Figure 2 shows an example of the time evolution of the tailless waterbag core simulated with the PIC code "WARP" [54]. The initial distribution has been well matched to the lattice, including the Debye screening effect [55]. When the operating point is inside the third-order stop band of $\left(k, \ell, n^{\prime}\right)=(3,0,1)$ predicted by the condition (8), a strong core deformation into a trianglelike shape is observed indicating the instability of the sextupole collective mode. Note that the bare tune is far from $1 / 3$ as opposed to the common understanding about the third-order resonance. At an operating point within the stop band of $\left(k, \ell, n^{\prime}\right)=$ $(4,0,1)$, the core is distorted into a rectanglelike configuration, followed by the development of four arms around it. The bare tune is now near $1 / 8$ instead of $1 / 4$. Such core dynamics cannot be described by any model based on a rigid distribution function.

The incoherent picture as sketched in Fig. 1, rather than the more accurate self-consistent picture, has been adopted universally to look for an optimum machine operating point. One reason why is that the FSM or the concept of incoherent tune spread is easy-to-use. Another clear advantage is that the FSM-based computation is much faster than self-consistent simulations. A question is how accurately such an approximate model without self-consistency reflects the realistic core resonance process. It is often said that two essentially different resonance mechanisms, "coherent" and "incoherent", exist simultaneously in a beam core. The incoherent mechanism is supposed to survive even after the coherent motion is somehow damped away. However, the core stability analysis based on the selfconsistent set of equations predicts no resonance under the incoherent condition. Assuming that the Vlasov theory covers the whole relevant physical processes, the most reasonable conclusion should be that no serious resonance occurs within the matched beam core at the incoherent tunes $\left(\nu_{x}, \nu_{y}\right)$ of individual particles. Numerical examples in Appendix A support this expectation. A similar conclusion has been reached previously in Ref. [30] and, for 


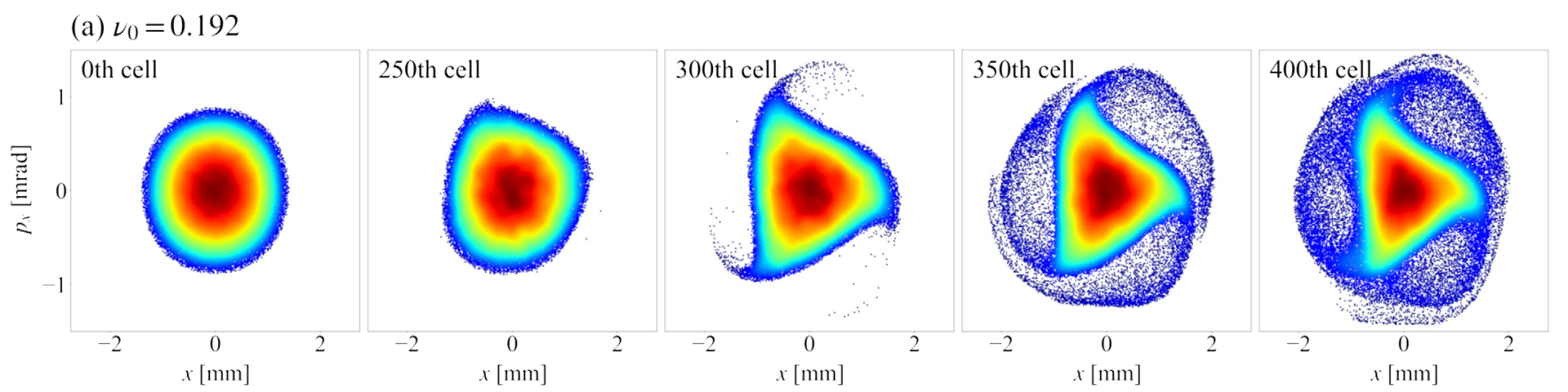

(b) $\nu_{0}=0.147$
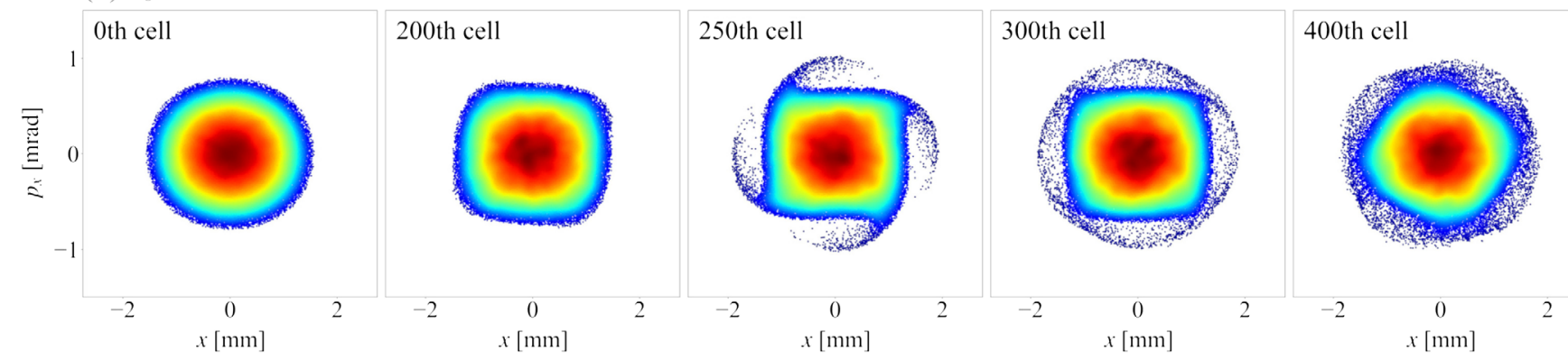

FIG. 2. Results of 2D WARP simulations. We have assumed a proton beam propagating through the symmetric sinusoidal focusing channel at the kinetic energy of $1 \mathrm{MeV}$. The external force is perfectly linear (no error fields). The initial distribution is the waterbag-type matched to the lattice. The tune depressions are adjusted to $\eta_{x}=\eta_{y}=0.8$ at the beginning. The ordinate represents the horizontal kinetic momentum normalized with the design value. The transverse bare tunes have been set equal $\left(\nu_{0 x}=\nu_{0 y}\right)$ and chosen to be (a) $\nu_{0}=0.192$ and (b) $\nu_{0}=0.147$. According to the coherent resonance condition in Eq. (7), the operating point is located within the sextupole $(m=3)$ stop band in the former case and within the octupole $(m=4)$ stop band in the latter.

the linear mode, in Ref. [19]. If all collective resonances are Landau-damped, then the core will be stable.

A sort of incoherent effect can be manifested once the coherent resonance or some other instability mechanism destroyed the initial distribution developing a complex coreand-tail structure. Such resonance-induced halo formation from an initially matched beam has been studied, e.g., by Holmes et al. [56]. As briefly remarked above, the beam halo is an issue that should be distinguished from the core resonance. We need to be careful not to confuse the core stability issue with tail resonance issues.

The PCM is a popular theoretical approach to halo formation problems. For more accurate information about what happens after the matched beam core is strongly distorted, we need to solve the Vlasov equation nonperturbatively or do self-consistent numerical simulations. The particles contributing to the thin tail formation usually circulate around the distorted core in phase space with large amplitudes. Since the coupling with the dense core is weak in the tail region, these halo particles will act more or less in an incoherent way. They resonate with the lattice or with the oscillating core space-charge potential near the incoherent tunes. We expect this type of quasi-single-particle resonance to be enhanced especially when the external driving force is present. If this happens, the widening of an instability band toward the single-particle $(\eta=1)$ resonance line is observed as discussed later in Sec. IV.

\section{STOP BANDS IN AN IDEAL AG LATTICE}

We now try to examine whether the coherent resonance conjecture in Eq. (8) can predict the approximate positions of low-order resonance lines in the tune diagram. The WARP code is employed for this purpose. The precise estimation of the tune-shift factor $C_{m}$ is, however, not so easy because of several reasons. First, all stop bands shift gradually during the progress of resonant instability that inevitably causes beam-density reduction. This makes it difficult to spot the original stop-band locations. Second, the beam tail (halo) starts to develop in some cases after the core becomes unstable. This side effect gives rise to stopband broadening or even splitting that affects the numerical procedure for estimating the central position of the resonance. Once it happens, the coherent effect on the stopband shift is overestimated; namely, we may conclude an inaccurate $C_{m}$-factor significantly smaller than the real value because the tail particles have smaller incoherent tune shifts than the core particles. Third, the $m$ th-order resonance band can overlap with higher-order resonance bands. This also worsens the accuracy of the band-shift evaluation for a particular mode. All these points complicate the process of the numerical determination of the $C_{m}$-factor, but physically most important here is to show that the observed stop-band locations cannot be explained by the incoherent resonance condition with a typical incoherent tune spread. 
We search for instability regions in the tune diagram with the WARP code, starting from the Gaussian distribution. The sinusoidal focusing force is assumed for simplicity. As numerically and experimentally verified in our previous work [31,35], the sinusoidal lattice has substantially the same resonance characteristics as the most standard FODO lattice. No imperfection fields are considered in this section, which guarantees that all nonlinear resonances identified are purely self-field-driven. Three different matching conditions are imposed initially to clarify the $\eta$-dependence of stop bands. While we mostly assume $1 \mathrm{MeV}$ proton beams in the present PIC simulations, neither the kinetic energy nor particle species are essential; in fact, the coherent resonance condition does not explicitly contain these parameters. The rms emittance growth at a particular operating point depends only on the rms tune depressions.

Throughout the paper, "emittance growth" is defined by subtracting unity from the ratio of the rms emittance after a certain AG periods to its initial value. It is given as a percentage and calculated separately in the horizontal and vertical directions rather than taking the average. That is not only because the initial rms emittances are not always equal in both directions but also due to the fact that the emittance exchange occurs under coupling resonances. In the tune diagrams and other color-coded pictures that appear in the following sections, we have taken the horizontal or vertical growth, whichever is greater, to choose the color.

\section{A. Stop bands with fixed tune depression}

Let us first study the case where the horizontal and vertical tune depressions are equal and fixed over the whole tune space. If the conjecture in Eq. (8) is valid, all resonance lines become straight in the tune diagram. Substitution of $\eta_{x}=\eta_{y}(\equiv \eta)$ into Eq. (8) leads to

$$
k \nu_{0 x}+\ell \nu_{0 y}=\frac{n^{\prime}}{2} \cdot \frac{1}{1-(1-\eta) C_{m}}
$$

that expresses a straight line on the $\nu_{0 x}-\nu_{0 y}$ plane. Figure 3 summarizes the results of systematic WARP simulations performed at five thousand different operating points with the tune depression fixed to $\eta=0.9$. All visible instability bands look straight. The linearity of stop bands has been confirmed for several other choices of $\eta$. The three particularly wide instability bands where serious emittance growth is detected should be attributed to the lowest-order resonance of the quadrupole mode with $\left(k, \ell, n^{\prime}\right)=(2,0,1)$, $(0,2,1)$, and $(1,1,1)[48,57]$. The dipole $(m=1)$ resonance cannot be excited under the lattice condition considered here. Although the fourth $(m=4)$ or higher-order coherent instabilities can overlap, they are much weaker than the linear instability according to the Vlasov theory.

Not surprisingly, the linear difference resonance with $\left(k, \ell, n^{\prime}\right)=(1,-1,0)$ is almost invisible in Fig. 3 and even in S-POD experiments [31]. This is because the horizontal

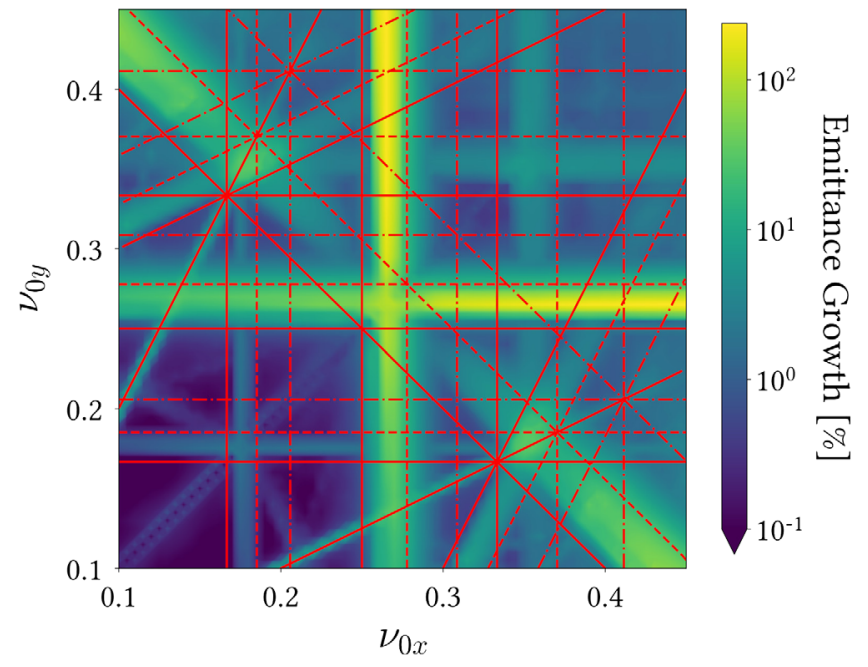

FIG. 3. Tune diagram obtained from 2D WARP simulations under the initial condition $\eta_{x}=\eta_{y}=0.9$. The rates of $\mathrm{rms}$ emittance growth evaluated at 5000 different operating points after 100 AG cells are color-coded in the tune diagram. The external beam-focusing force is completely linear, which varies sinusoidally along the transport channel. The abscissa and ordinate represent the horizontal and vertical bare tunes per AG cell. The initial particle distribution is the Gaussian type well matched the AG potential. The solid, dashed, and dash-dotted lines in the picture are obtained from Eq. (10) with the incoherent tune shifts $\Delta \nu_{x(y)}=0, \Delta \bar{\nu}_{x(y)}$, and $\max \left(\Delta \nu_{x(y)}^{\text {Gauss }}\right)$, respectively. We have assumed that all visible emittance-growth bands are due to the linear $(m=2)$ and first nonlinear $(m=3)$ resonances. Two of the four third-order coupling resonance lines are independent of the $C_{m}$ factor because they correspond to the $n^{\prime}=0$ case in Eq. (9). A scalloped appearance of these coupling resonance bands is due to the limited density of PIC data points.

and vertical rms emittances $\left(\varepsilon_{x}, \varepsilon_{y}\right)$ are equal in a matched beam along the line $\nu_{0 x}-\nu_{0 y}=0$. No emittance exchange mechanism is activated in such an isotropic beam on the symmetric difference resonance. This observation is consistent with the typical operating condition of a highintensity hadron linac where the horizontal and vertical betatron phase advances (bare cell tunes) are mostly set equal or close to each other. It is possible to demonstrate experimentally, by the use of initially anisotropic ion plasmas in S-POD, that the coupling resonance does exist on $k \nu_{0 x}-k \nu_{0 y}=0$. Typical measurement data are presented in Appendix B.

In addition to the three quadrupole resonance lines, we find eight more instability bands in Fig. 3, all of which can be explained as the lowest-order nonlinear $(m=3)$ resonance with $\left(k, \ell, n^{\prime}\right)=(3,0,1),(0,3,1),(3,0,2),(0,3,2)$, $(2,-1,0),(-1,2,0),(2,-1,1)$, and $(-1,2,1)$ [58]. These nonlinear stop bands except for the last two have been observed even experimentally as reported in Ref. [31]. It is also possible, by improving the sensitivity to emittance variation, to confirm the existence of very low but nonzero emittance growth along the third-order sum resonance lines 
with $\left(k, \ell, n^{\prime}\right)=(2,1,1),(1,2,1),(2,1,2)$, and $(1,2,2)$. We can detect very weak coupling effect slightly above and below the line $\nu_{0 x}-\nu_{0 y}=0$ as well. Under the initial condition taken in this subsection, the horizontal and vertical rms emittances are unequal except along this symmetry line. The small emittance imbalance in the close vicinity triggers the faint difference resonance. The observed stop bands now cover all possible combinations of the three integers $\left(k, \ell, n^{\prime}\right)$ for the linear $(m=2)$ and first nonlinear $(m=3)$ coherent resonances. If we ignore the parametric factor $1 / 2$ on the right-hand side of Eq. (9), the origin of the stop bands with odd $n^{\prime}$ has to be interpreted as the nonlinear resonances of twice the orders, i.e., the fourth $(m=4)$ and sixth $(m=6)$ orders. Then it seems difficult to provide a rational explanation for many missing fourth-order and sixth-order resonance lines. Moreover, no fifth-order resonance bands have been detected so far in the absence of external error fields.

As is clear from the numerical data in Appendix A (see Fig. 20), the rms tune shift $\Delta \bar{\nu}$ is much smaller than the maximum size of the incoherent tune shift $\Delta \nu$ in the Gaussian beam. For reference, in Fig. 3 we have drawn the resonance lines defined with the incoherent tune shifts $\left(\Delta \nu_{x}, \Delta \nu_{y}\right)$ by

$\Gamma_{k \ell}\left(\Delta \nu_{x}, \Delta \nu_{y}\right) \equiv k\left(\nu_{0 x}-\Delta \nu_{x}\right)+\ell\left(\nu_{0 y}-\Delta \nu_{y}\right)=\frac{n^{\prime}}{2}$.

Solid lines correspond to the case where both tune shifts are zero, i.e., $\Gamma_{k \ell}(0,0)=n^{\prime} / 2$. This is identical to the single-particle resonance condition in Eq. (1) except for the $1 / 2$ factor on the right-hand side. Dashed lines represent $\Gamma_{k \ell}\left(\Delta \bar{\nu}_{x}, \Delta \bar{\nu}_{y}\right)=n^{\prime} / 2$ indicating where the coherent resonance bands should be if $C_{m}=1$. Dashdotted lines are obtained by taking the maximum incoherent tune shifts in the Gaussian distribution, i.e., $\Gamma_{k \ell}\left(\max \left(\Delta \nu_{x}^{\text {Gauss }}\right), \max \left(\Delta \nu_{y}^{\text {Gauss }}\right)\right)=n^{\prime} / 2$. These Gaussian tune shifts can be estimated roughly from Eq. (A3) in the Appendix A. According to the common understanding based on the incoherent picture as in Fig. 1, we are not allowed to put the machine operating point inside the area between the solid and dash-dotted lines. It is, however, evident from Fig. 3 that such a stability criterion is too conservative. Furthermore, all observed stop bands lie inbetween the corresponding solid and dashed lines. This implies that the effective shift of each band is somewhat smaller than the rms tune shift. It is also worth noting that the band widths depend on the resonance orders, which cannot be explained by the incoherent picture in Fig. 1 . These facts can be regarded as evidence that the core resonance is described not by the incoherent condition in Eq. (3) with a large tune spread but by the coherent condition in Eq. (9) with the tune-shift factor of the range $0<C_{m}<1$. More discussion on the ambiguity of the incoherent picture has been made in Appendix A. We shall try to estimate the $C_{m}$-factors of low-order modes in Sec. IV by introducing external driving perturbations.

\section{B. Stop bands with fixed beam intensity and emittance}

The beam current and emittances are usually given at injection, depending on the performance of an ion source, pre-accelerators, etc. When these parameters are kept constant regardless of the machine operating point, the initial tune depressions $\eta_{x(y)}$ become a function of the bare tunes. The resonance lines defined by Eq. (8) are then no longer straight. We conducted WARP simulations taking this practical situation into account. The emittance growth after 100 AG periods are color-coded in the tune diagram of Fig. 4 where the initial current and rms emittances have been both fixed to specific values over the whole tune space. The horizontal and vertical emittances are set equal $\left(\varepsilon_{x}=\varepsilon_{y}\right)$, assuming a round beam from an ion source. The beam intensity is chosen so that $\eta_{x}$ and $\eta_{y}$ take a given value $\left(\equiv \eta_{1 / 6}\right)$ at the operating point $\left(\nu_{0 x}, \nu_{0 y}\right)=(1 / 6,1 / 6)$. The tune diagram in Fig. 4 is the case where $\eta_{1 / 6}=0.9$. This corresponds, for example, to about $30 \mathrm{~mA}$ of a $10 \mathrm{MeV}$ proton beam with the normalized rms emittance of $0.1 \pi \mathrm{mm} \cdot \mathrm{mrad}$ when the unit AG cell is $1 \mathrm{~m}$ long. All major resonances discovered in Fig. 3 have been excited in Fig. 4. In addition to them, we notice the existence of very weak fourth-order resonance lines with $\left(k, \ell, n^{\prime}\right)=(4,0,3)$ and $(0,4,3)$. Each stop band still looks straight because the tune-dependent variation of $\eta_{x(y)}$ is not so significant at this intensity.

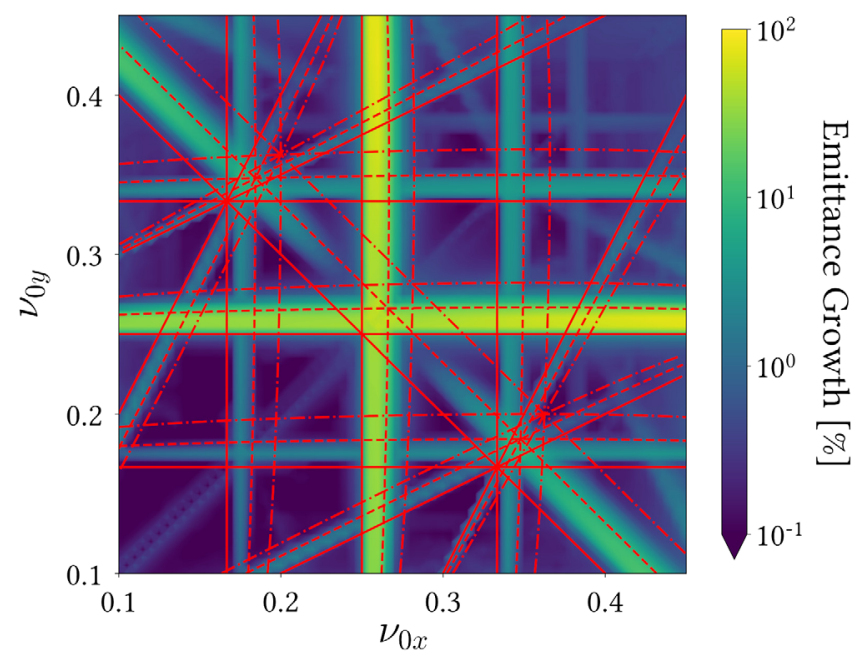

FIG. 4. Tune diagram obtained from 2D WARP simulations with a fixed beam intensity and fixed rms emittances at injection. The initial intensity and emittances are maintained everywhere on the diagram at the values that give $\eta_{x}=\eta_{y}=0.9$ at the operating point $\left(\nu_{0 x}, \nu_{0 y}\right)=(1 / 6,1 / 6)$. The tune depressions are no longer constant but vary depending on the cell tunes. Other numerical conditions and the definitions of the red lines are the same as in Fig. 3. 


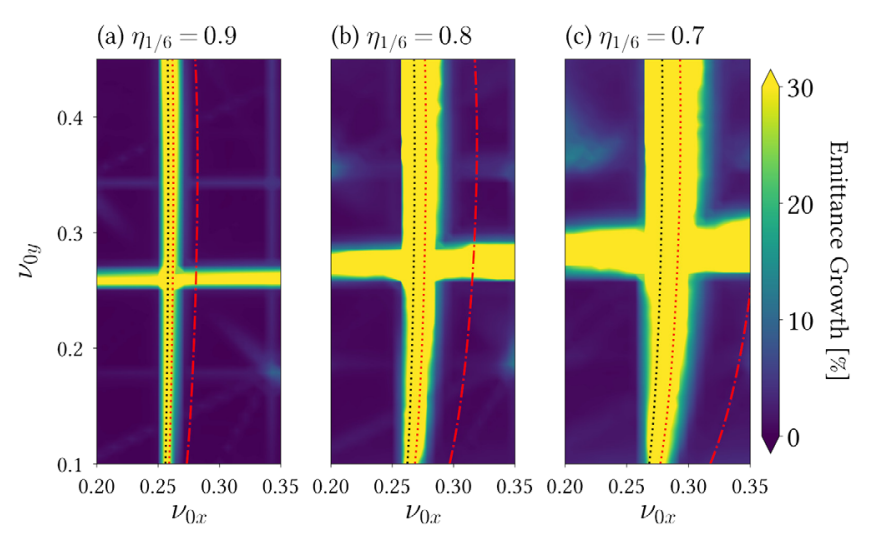

FIG. 5. Beam-intensity dependence of the stop band near $\nu_{0 x}=1 / 4$ obtained from 2D WARP simulations. The initial beam parameters are determined in the same way as in Fig. 4. The same color has been used in the region where the emittance growth exceeds $30 \%$. The tune depressions at the operating point $\left(\nu_{0 x}, \nu_{0 y}\right)=(1 / 6,1 / 6)$ are adjusted to (a) $\eta_{1 / 6}=0.9$, (b) $\eta_{1 / 6}=0.8$, and (c) $\eta_{1 / 6}=0.7$. The dotted lines are the theoretical prediction from Eq. (8) for the linear $(m=2)$ resonances associated with the quadrupole mode (red) and breathing mode (black). The tune-shift factors have been assumed to be $C_{2}=0.75$ for the former mode and $C_{2}=0.5$ for the latter. There seem to be no resonances exactly along those lines. The dash-dotted line in each panel represents $\Gamma_{20}=1 / 2$ with the maximum incoherent tune shift of the Gaussian distribution.

The effect of varying tune depression can be made clear by increasing the initial beam current. The most severe stop band above $\nu_{0 x}=1 / 4$ is plotted in Fig. 5 at three different currents corresponding to $\eta_{1 / 6}=0.9,0.8$, and 0.7. A slight bending of the stop band is now recognizable at higher beam density. The dotted lines in each panel are derived from the 2D resonance conjecture for the linear $(m=2)$ modes with $\left(k, \ell, n^{\prime}\right)=(2,0,1)$. $C_{2}$ has been adjusted to 0.75 (red) and 0.5 (black). These numbers come from the rms envelope equations that describe possible collective oscillations with elliptical symmetry [50]. The former number corresponds to the quadrupole mode and the latter to the so-called breathing mode. Although both curves are inside the emittancegrowth band, we observe no clear signature of the two different types of instabilities overlapping. It is possible to show, by numerically integrating the rms envelope equations, that this linear stop band actually has a single peak of emittance-growth rate along a line above $\nu_{0 x(0 y)}=1 / 4$. The peak position can be best fitted with $C_{2}$ slightly below 0.75 (cf. Table I in Sec. IV). Even experimentally (see, e.g., Fig. 24 in Appendix B and Ref. [31]), no second peak has been found in a linear stop band, except for the one generated by the coherent dipole $(m=1)$ mode [39]. The breathing mode does not appear to be excited seriously in a quadrupole channel. From a practical point of view, therefore, it is allowed to assign a single value to $C_{2}$.

\section{Stop bands of the initially equipartitioned beam}

Another initial condition physically interesting to us is the equipartitioned case where the beam at injection satisfies $[10,53,59]$

$$
\frac{\varepsilon_{x}}{\varepsilon_{y}}=\frac{\eta_{y} \nu_{0 y}}{\eta_{x} \nu_{0 x}} .
$$

The tune diagram in Fig. 6 is obtained with the initial particle distribution constructed under this condition. The beam perveance has been fixed to the same value as adopted in Fig. 4. The linear resonance bands are slightly curved toward the direction opposite to the previous case of fixed intensity and emittance. At higher beam density, the curvature is more enhanced as displayed in Fig. 7. The 2D resonance conjecture in Eq. (8) has again succeeded in reproducing the basic feature of the low-order stop band distribution.

Interestingly, two of the third-order difference resonance bands identified in Figs. 3 and 4 have almost disappeared in Fig. 6. They do not manifest themselves even if a thirdorder external potential is added to the lattice. Figure 8(a) shows an example when the sextupole error field proportional to $x^{3}-3 x y^{2}$ is switched on. The difference resonance line is still invisible. The contour plot in Fig. 8(b) suggests an underlying physical factor behind this resonance suppression. We recognize that the emittance-based quantity

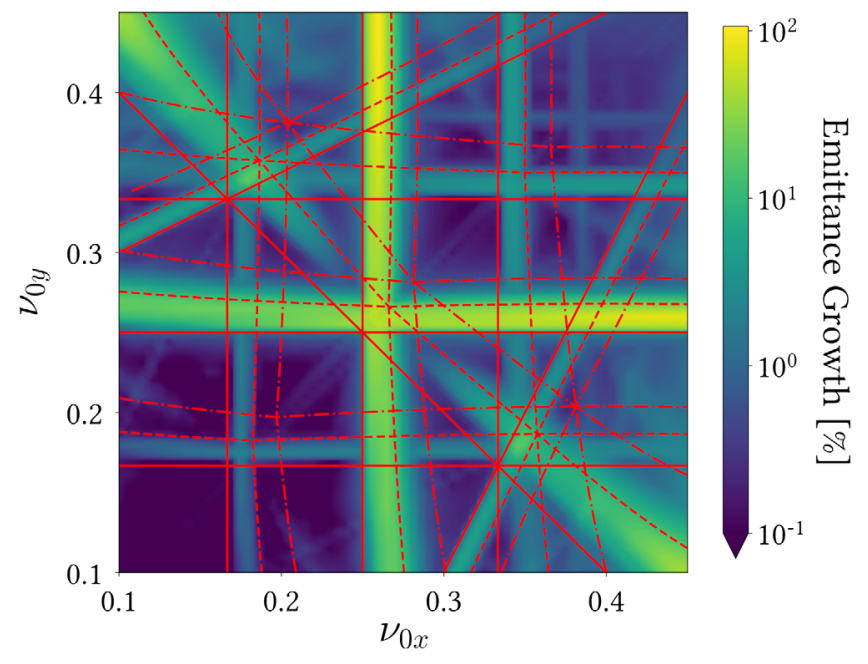

FIG. 6. Tune diagram obtained from 2D WARP simulations with the initially equipartitioned beam. The beam intensity has been fixed to the value that gives $\eta_{x}=\eta_{y}=0.9$ at the operating point $\left(\nu_{0 x}, \nu_{0 y}\right)=(1 / 6,1 / 6)$. The initial rms emittances $\varepsilon_{x(y)}$ at each operating point are determined such that the requirement in Eq. (11) is met. Other numerical conditions and the definitions of the red lines are the same as in Fig. 3. Note that the third-order difference resonances with $\left(k, \ell, n^{\prime}\right)=(2,-1,0)$ and $(1,-2,0)$ have been extremely weakened. 


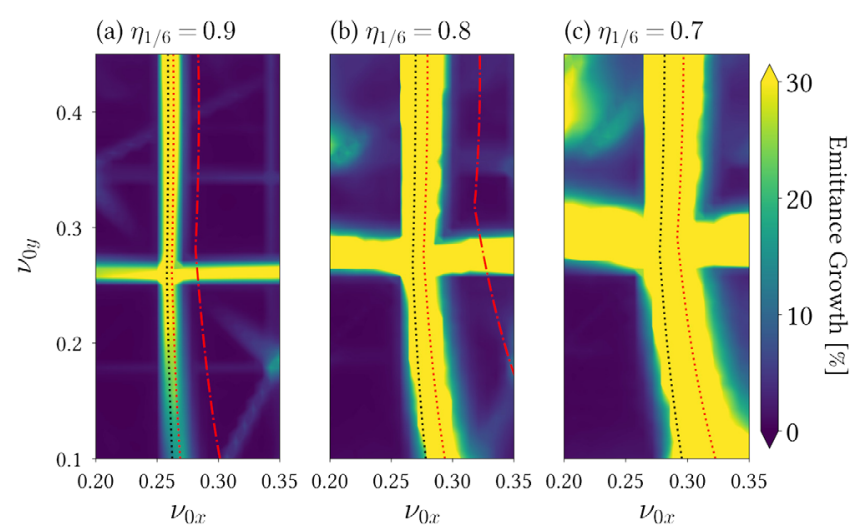

FIG. 7. Beam-intensity dependence of the stop band near $\nu_{0 x}=$ 1/4 obtained from 2D WARP simulations under the equipartitioning condition. Similarly to the examples in Fig. 5, we have considered the three different beam intensities corresponding to (a) $\eta_{1 / 6}=0.9$, (b) $\eta_{1 / 6}=0.8$, and (c) $\eta_{1 / 6}=0.7$. The definitions of the red and black dotted lines are the same as in Fig. 5.

(a) Emittance Growth [\%]

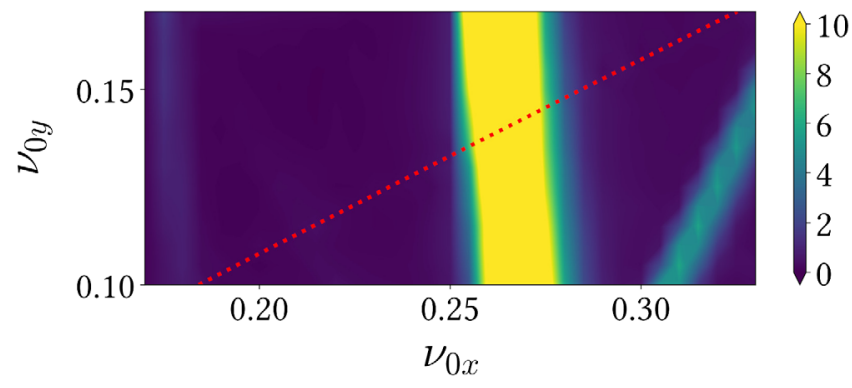

(b) $I_{k \ell}$

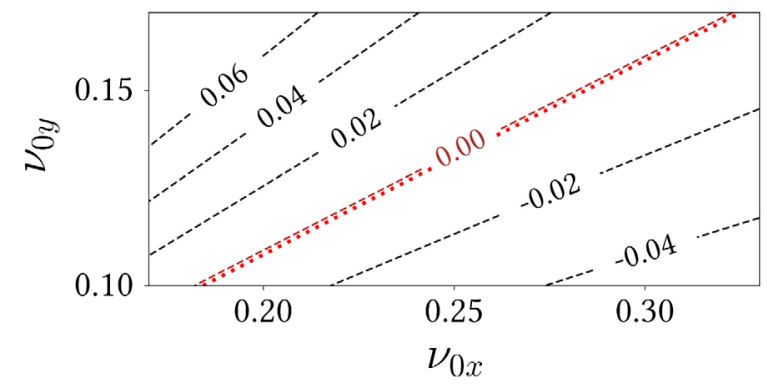

FIG. 8. PIC results on the suppression of a third-order difference resonance line. (a) Tune diagram based on WARP simulations with the initially equipartitioned Gaussian beam. The simulation parameters are the same as employed in Fig. 6 except that we have now switched a third-order error field $\left(\propto x^{3}-3 x y^{2}\right)$ on. The amplitude of the nonlinear external field is one percent of the linear focusing field's. (b) Contour plot of the quantity $I_{k \ell}$ defined by Eq. (12) under the equipartitioning condition. The numbers on the picture indicate the values of $I_{k \ell}$ in units of $\mathrm{mm} \cdot \mathrm{mrad}$. The red dotted line is the position of the coherent resonance band predicted by Eq. (8) with $\left(k, \ell, n^{\prime}\right)=(1,-2,0)$. If we assume that $C_{3}=0.875$ [20], the coherent resonance band predicted by Eq. (8) is positioned along the red dotted line that almost perfectly agrees with the line of $I_{k \ell}=0$. This supports the theoretical expectation that the coherent tune-shift factor is smaller than unity.

$$
I_{k \ell} \equiv \frac{\varepsilon_{x}}{k}+\frac{\varepsilon_{y}}{\ell}
$$

has become exactly zero along the missing third-order difference $(k \ell<0)$ resonance line corresponding to $\left(k, \ell, n^{\prime}\right)=(1,-2,0) . I_{k \ell}$ is very similar to the well-known invariant $\tilde{I}_{k \ell} \equiv \varepsilon_{x} / k-\varepsilon_{y} / \ell$ on the incoherent sum and difference resonances driven by the coupling term $x^{|k|} y^{|\ell|}$ $[2,3,60]$. The condition $I_{k \ell}=0$ has been fulfilled along the other missing third-order line with $\left(k, \ell, n^{\prime}\right)=(2,-1,0)$ and also along the difference resonance $\nu_{0 x}-\nu_{0 y}=0$ hardly detectable in all three cases studied in Sec. III. Another piece of evidence is given in Appendix C where the emittance exchange along the difference resonance lines with $\left(k, \ell, n^{\prime}\right)=(2,-1,0)$ and $(2,-1,1)$ has been eliminated successfully by imposing the condition $\varepsilon_{x}=2 \varepsilon_{y}$ on the initial emittances. The present findings about difference resonances should apply to the beam of any density $(0<\eta<1)$ as Eq. (12) is free from intensitydependent parameters.

\section{STOP BANDS IN THE PRESENCE OF EXTERNAL PERIODIC PERTURBATION}

In the 1D case, the external-field-driven and self-fielddriven resonance conditions take the same form except for the parametric factor $1 / 2$, provided that external nonlinearity can be treated as weak perturbation [27]. The tune-shift constant $C_{m}$ is independent of the origin of the driving force. We have conjectured in Eq. (8) that this theoretical prediction for a $1 \mathrm{D}$ beam holds in the 2D case as well. The numerical estimation of $C_{m}$ can then be achieved by introducing an artificial driving potential. In this section, we switch on a low-order external perturbing field in addition to the sinusoidal focusing field. Since we do not rely on the natural Coulomb potential any more, the resonance strength of a specific order is controllable independently of the tune depression. Furthermore, in computer simulations any periodicity is available for the perturbing field, unlike in a real storage ring where external imperfections normally repeat every turn. The right-hand side of the external-field-driven resonance condition is, therefore, not necessarily an integer $n$ but allowed to take an arbitrary number [see Eq. (13)]. It has been demonstrated experimentally that random noise on the AG focusing field in a circular accelerator may induce this type of resonance and possibly affects the long-term stability of intense hadron beams [34,61].

We expect the resonance condition (8) to hold for any matched beam distributions with the same values of $C_{m}$ 's. Equation (8) actually depends only on the rms tune shifts that are insensitive to the distribution function of particles in phase space [50]. In addition to the Gaussian model, we here consider the waterbag and parabolic models to check whether the choice of a different distribution function may cause significant change in the size of the estimated $C_{m}$. 


\section{A. Noncoupling resonances}

The OY theory suggests that the use of the $m$ th-order perturbing field of the frequency $f_{m}$ makes it possible to excite the coherent resonance of a particular transverse direction $(x$ or $y)$ under the condition

$$
m\left(\nu_{0}-C_{m} \Delta \bar{\nu}\right)=\left|n \pm \kappa_{m}\right|,
$$

where $\kappa_{m}$ is the ratio of the driving frequency $f_{m}$ to the linear focusing-field frequency $f_{0}$; namely, $\kappa_{m}=f_{m} / f_{0}$. The existence of this type of resonance has been confirmed experimentally with S-POD [34]. A nonlinear driving perturbation can yield even more stop bands other than those under the condition in Eq. (13), but in the following, we focus on the most severe stop band at

$$
m \nu_{0}=\frac{\kappa_{m}}{1-(1-\eta) C_{m}}
$$

corresponding to the $n=0$ case. A major advantage of using this external-field-driven stop band is the absence of overlapping resonances of different orders. We can locate the $m$ th-order resonance band by varying the perturbation frequency rather than the operating tune. Given $\nu_{0}$ and $\eta$, the coherent mode of a higher $m$ number resonates at a higher $\kappa_{m}$. For example, the octupole resonance occurs at the perturbation frequency twice higher than the value at which the quadrupole resonance is excited. The two stop bands plotted as a function of $\kappa_{m}$ are thus well separated. In this subsection, we keep the operating point at $\left(\nu_{0 x}, \nu_{0 y}\right)=$ $(0.15,0.15)$ while scanning the perturbation frequency. The horizontal and vertical tune depressions are set equal, i.e., $\eta_{x}=\eta_{y}(\equiv \eta)$.

Before proceeding to the $C_{m}$ estimation, we take a brief look at the role of the Gaussian tail that may obscure the position of a core resonance band. Figure 9(a) shows the emittance behavior of Gaussian beams driven by the second-order perturbation. The emittance growth is calculated at the exit of the 200th sinusoidal focusing cell. The tune depression is adjusted initially to $\eta=0.9$ (black) and
0.8 (red). The external-field-driven linear stop bands of waterbag and parabolic beams are exhibited in Figs. 9(b) and 9(c) for comparison. The sawtooth-like configuration of the emittance-growth curves in the waterbag and parabolic cases are well known and has been observed repeatedly in S-POD experiment [31,35-41]. It is formed because the beam stays in a resonance band for a longer period at a larger $\kappa_{2}$, thus experiencing more emittance growth. The emittance growth results in the reduction of the beam's phase-space density, which makes the tune depression approach unity. The resonant value of $\kappa_{2}$ in Eq. (14) then becomes larger when $\nu_{0}$ is fixed; in other words, the stop-band's center moves toward the higher- $\kappa_{2}$ side in Fig. 9 during the emittance growth process.

The Gaussian stop band in Fig. 9(a) has a unique feature obviously different from the other two. Specifically, it is much wider and extended toward the upper threshold $\left(\kappa_{2}=0.3\right)$ given by the single-particle resonance condition $2 \nu_{0}=\kappa_{2}$. We even observe a double-peak configuration when $\eta=0.9$. No such extra peak has appeared in the $\eta=0.8$ case, but we see the growth rate gently decreasing on the higher- $\kappa_{2}$ side, which considerably broadens the instability region. The double-peak profile in Fig. 9(a) is not the consequence of the theoretically predicted complexity of the coherent tune-shift factor [25,30,47]. It also has nothing to do with the double stop-band structure reported in Ref. [39], which is formed near every half-integer tune by the overlapping of two coherent resonances associated with the dipole $(m=1)$ and quadrupole $(m=2)$ oscillation modes. We have no such overlapping with the strong dipole mode here.

The peculiarity of the Gaussian distribution originates largely from the existence of the long tail where the Coulomb coupling with the beam core is weak. The motions of individual particles far from the core are no longer strongly correlated and, therefore, could be affected under the condition (3) with relatively small tune shifts. As these tail particles gain larger amplitudes in the progress of instability, their tune shifts come closer to zero. The (a) Gaussian

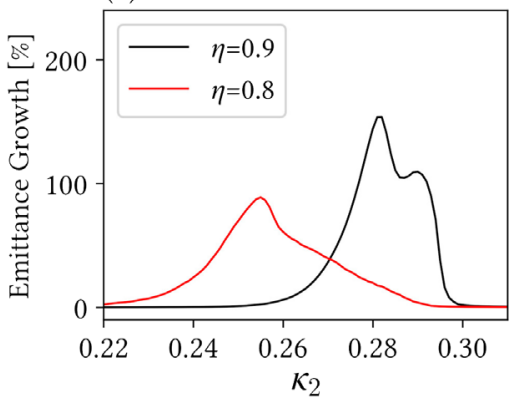

(b) Waterbag

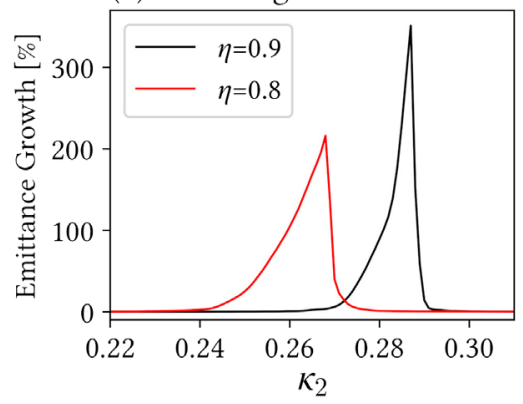

(c) Parabolic

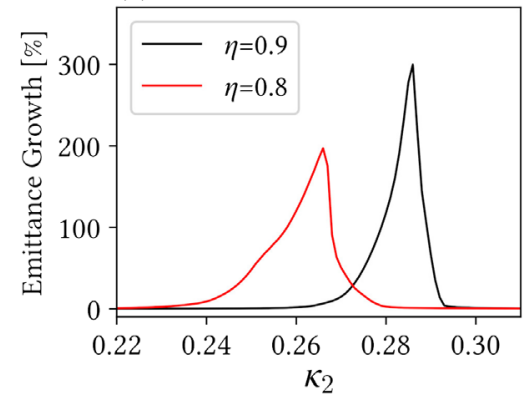

FIG. 9. Stop-band configurations after beam transport over 200 sinusoidal focusing cells. The bare tunes have been fixed to 0.15 in both transverse directions $\left(\nu_{0}=0.15\right)$. The external linear perturbation of the driving tune $\kappa_{2}$ is applied to excite the coherent quadrupole mode. The perturbation amplitude is set at $0.4 \%$ of the focusing field's. The ordinate represents the average transverse rms emittance growth at the 200th cell, plotted as a function of $\kappa_{2}$. Three different types of initially matched distributions with $\eta=0.8$ and 0.9 are considered: (a) Gaussian, (b) waterbag, and (c) parabolic. 

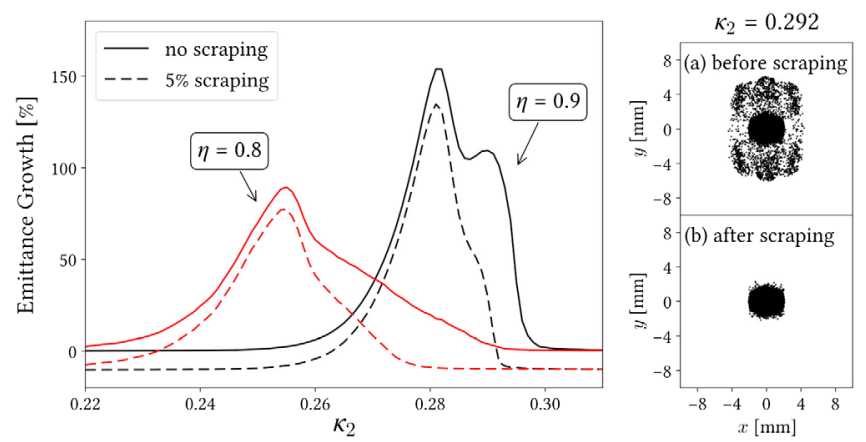

FIG. 10. Stop-band configurations of a Gaussian beam before and after halo scraping. The stop band at the 200th sinusoidal cell in Fig. 9 is replotted with a solid line for comparison. The dashed line indicates the band profile after $5 \%$ of tail particles with large oscillation amplitudes in phase space are scraped off. The final cross-sectional views of the beam at $\kappa_{2}=0.292(\eta=0.9)$ before and after the scraping are shown in the right panels.

emittance growth of tail origin then arises near the singleparticle resonance line. Even though the particles in the tail are only a small portion of the whole beam, they give rise to non-negligible emittance growth because of their large oscillation amplitudes in phase space. The emittance-growth curves in Fig. 10 illustrate this fact. The solid line of the full Gaussian beam is altered to the dashed line by removing halo particles from the original distribution. Only five percent removal of outermost particles in four-dimensional phase space almost completely eliminates the second peak in the $\eta=0.9$ case. The halo scraping has also significantly lowered the emittance growth on the high- $\kappa_{2}$ side of the broad instability band in the $\eta=0.8$ case. In both cases, the highest peak is only weakly affected by the halo scraping procedure, which implies that it is created mainly by the core instability.
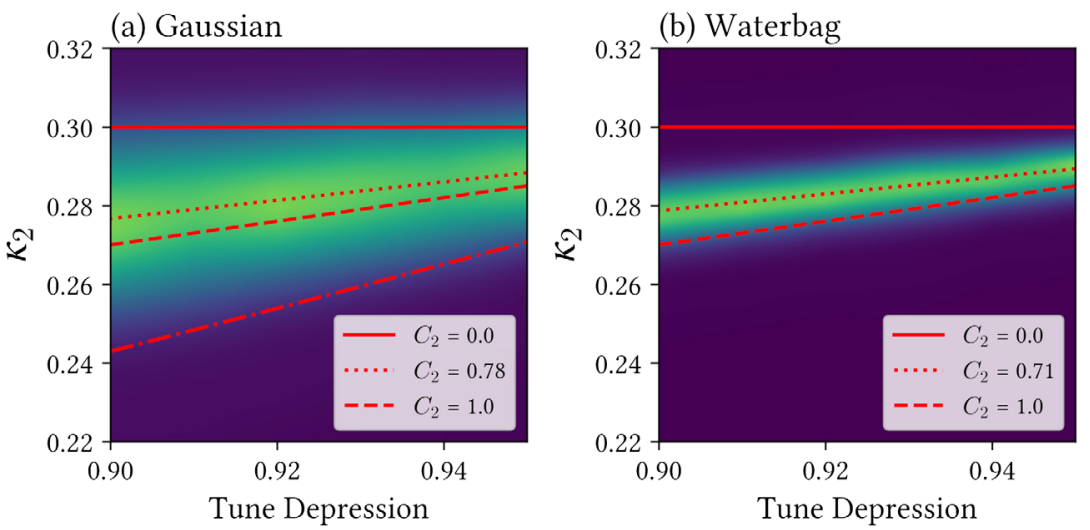

FIG. 12. Emittance-growth chart obtained from WARP simulations with the quadrupole driving perturbation on. Three different types of initial particle distributions, i.e., (a) Gaussian, (b) waterbag, and (c) parabolic, are considered, all of which are well matched to the sinusoidal focusing potential. The Gaussian tail has been truncated by one percent in four-dimensional phase space. The bare betatron tunes are fixed at $\nu_{0 x}=\nu_{0 y}=0.15$. The linear perturbation is applied to excite the quadrupole $(m=2)$ resonance. The perturbation amplitude is set at $0.2 \%$ of the focusing field's. The solid and dashed lines correspond to the cases where $C_{2}=0$ (single-particle resonance) and $C_{2}=1$. The dotted line is the location of the stop-band peak fitted by Eq. (14) with $C_{2}=0.78$ for Gaussian, $C_{2}=0.71$ for waterbag, and $C_{2}=0.73$ for parabolic. The dash-dotted line in the left panel indicates the incoherent resonance line for a particle with the maximum tune shift. 

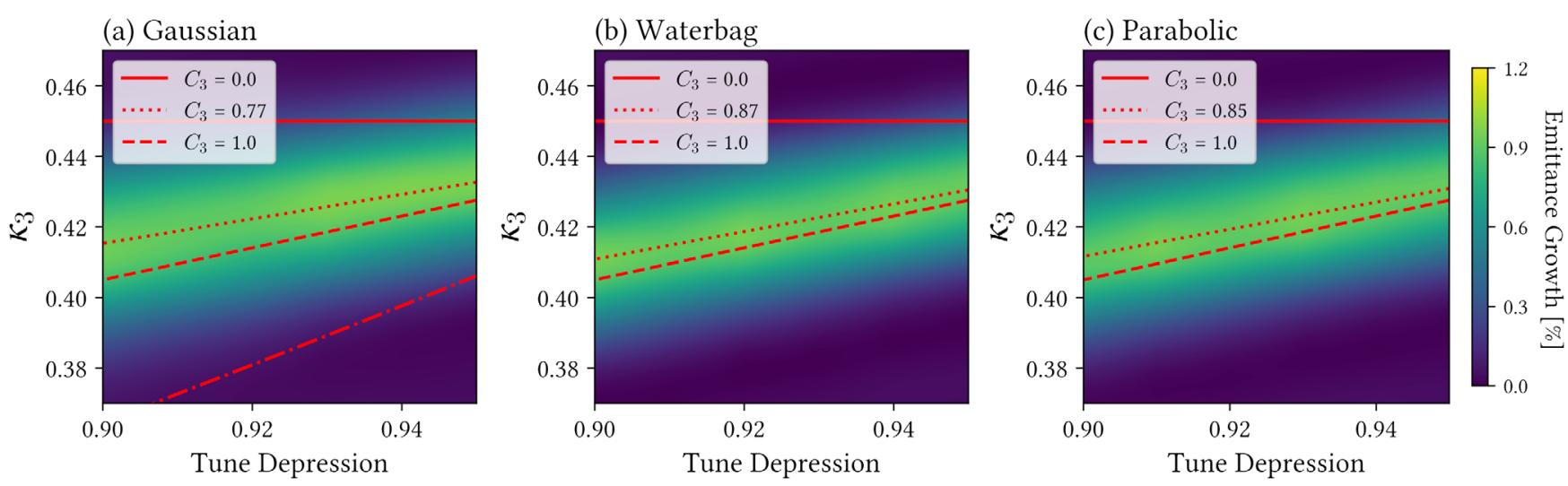

FIG. 13. Emittance-growth chart obtained from WARP simulations with the sextupole driving perturbation on. Except for the order of the external perturbing field, all numerical conditions are the same as employed in Fig. 12. The perturbation amplitude is set at $0.4 \%$ of the focusing field's. The dotted line is the location of the stop-band peak fitted by Eq. (14) with $C_{3}=0.77$ for Gaussian, $C_{3}=0.87$ for waterbag, and $C_{3}=0.85$ for parabolic.

halo particle grows, its incoherent tune approaches the bare tune $\nu_{0}$. The halo particle then becomes increasingly unstable and will eventually be lost when there exists any driving force that can excite the single-particle resonance under the condition $m \nu_{0} \approx \kappa_{m}$. An analogous beam-loss phenomenon in the vicinity of a single-particle resonance line has been observed experimentally at the CERN Proton Synchrotron [62]. The primary core resonance forms another peak on the lower side of the instability area in Fig. 11. It is located above the theoretically expected position indicated by the dotted line. The band shift is caused by the emittance growth that increases the tune depression toward unity.

For a better estimate of the $C_{m}$ factor, it is necessary to minimize the effect from halo formation as well as the stopband shift due to emittance growth. We here define the center of the $m$ th-order stop band as the point at which the peak of the emittance-growth curve plotted as a function of the perturbation tune $\kappa_{m}$ first exceeds the one-percent level. Since the emittance growth is still low, the band shift should be ignorable. We also expect no significant halo to be developed at this early stage of instability. In the Gaussian case, however, even a very small number of particles in the long tail may be a source of low-level emittance growth before the core becomes unstable. To mitigate such ambiguity, we truncate the Gaussian tail by one percent at the beginning.

The rms emittance growth evaluated with the WARP code are color-coded in Fig. 12 on $\eta-\kappa_{2}$ plane. These numerical observations under the quadrupole perturbation cannot be explained without the $C_{2}$-factor smaller than unity. The dash-dotted line in the left panel indicates the incoherent resonance line corresponding to the maximum Gaussian tune shift; namely, it is obtained from Eq. (13) by replacing the coherent tune-shift term $C_{m} \Delta \bar{\nu}$ by $\max \left(\Delta \bar{\nu}^{\text {Gauss }}\right) \approx\left(1-\eta^{2}\right) \nu_{0}$. The coherent tune-shift factor of the quadrupole mode estimated from these PIC simulations is $C_{2}=0.78 \pm 0.05$ for the Gaussian distribution, $C_{2}=0.71 \pm 0.04$ for the waterbag distribution, and $C_{2}=$ $0.73 \pm 0.05$ for the parabolic distribution. For reference, the coherent resonance condition in Eq. (14) is drawn with (a) Gaussian

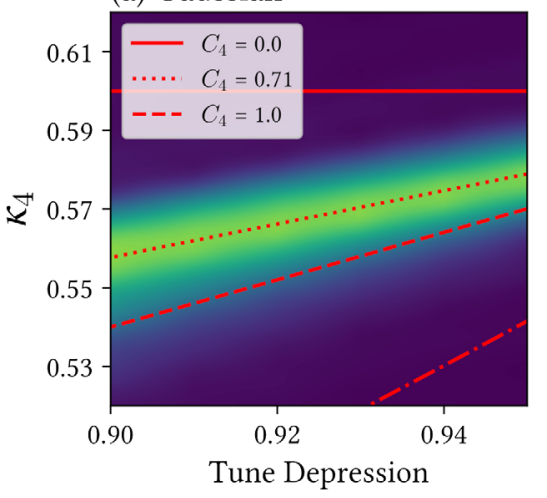

(b) Waterbag

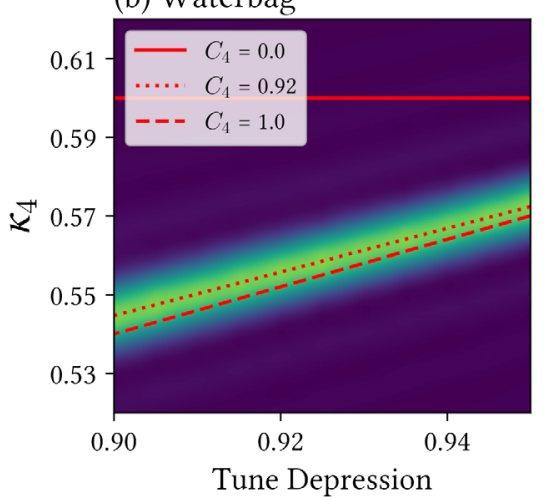

(c) Parabolic

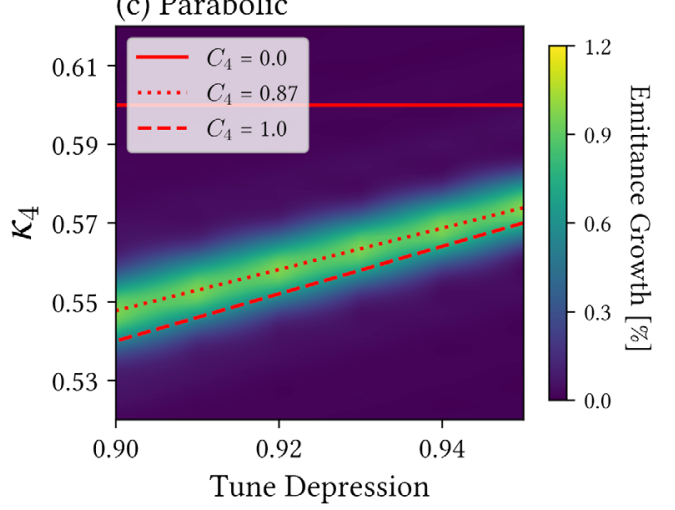

FIG. 14. Emittance-growth chart obtained from WARP simulations with the octupole driving perturbation on. Except for the order of the external perturbing field, all numerical conditions are the same as employed in Fig. 12. The perturbation amplitude is set at $0.6 \%$ of the focusing field's. The dotted line is the location of the stop-band peak fitted by Eq. (14) with $C_{4}=0.71$ for Gaussian, $C_{4}=0.92$ for waterbag, and $C_{4}=0.87$ for parabolic. 
TABLE I. Coherent tune-shift factors estimated from noncoupling resonance bands.

\begin{tabular}{lccc}
\hline \hline & $C_{2}$ & $C_{3}$ & $C_{4}$ \\
\hline Gaussian & $0.78 \pm 0.05$ & $0.77 \pm 0.06$ & $0.71 \pm 0.06$ \\
Waterbag & $0.71 \pm 0.04$ & $0.87 \pm 0.03$ & $0.92 \pm 0.01$ \\
Parabolic & $0.73 \pm 0.05$ & $0.85 \pm 0.04$ & $0.87 \pm 0.02$ \\
\hline \hline
\end{tabular}

a dotted line, assuming $C_{2}=0.78$ in Fig. 12(a), $C_{2}=0.71$ in Fig. 12(b), and $C_{2}=0.73$ in Fig. 12(c).

We did similar WARP simulations with a nonlinear driving perturbation on. Figures 13 shows the emittancegrowth chart when the weak normal sextupole $(m=3)$ field is activated. The peak position of the instability band can be fitted very well with the coherent resonance condition in Eq. (14). The tune-shift factor $C_{3}$ has to be less than unity for the best fitting in all three types of distributions. The stop band generated by the normal octupole $(m=4)$ perturbation is depicted in Fig. 14 from which we conclude $C_{4}<1$ as in the previous cases of the lower-order instabilities. No clear indication of incoherent resonance can be seen in Figs. 12, 13, and 14. The singlepeaked band profile in these figures suggests that, from a practical point of view, we only need a single tune-shift factor for each coherent mode of a specific order.

The fitting results of noncoupling coherent resonances driven by the external term proportional to $x^{m}(m=2,3$, and 4) are summarized in Table I. $C_{m}$ tends to be greater for a higher-order mode in the waterbag and parabolic cases as predicted by the 1D Vlasov theories [20,27]. The estimated central values of $C_{m}$ 's for the waterbag distribution are in excellent agreement with the Vlasov prediction; the Sacherer's analysis based on the uniform distribution says that $C_{2}=0.750, C_{3}=0.875$, and $C_{4}=0.922$.

The $C_{m}$-factor of the Gaussian beam is less than unity for any modes, but its dependence on the resonance order looks opposite to the other two cases. This must be due to remaining tail particles that expand the stop band toward the single-particle resonance line (cf. Fig. 9) and thus reduce the estimated value of $C_{m}$. In fact, we can make it larger by scraping more tail particles off initially. Too much initial scraping is, however, not preferable because it can enhance a mismatch to the lattice. The initial distribution generator of the WARP code has been designed on the basis of the theory in Ref. [55] assuming the full Gaussian distribution function.

\section{B. Coupling resonances}

According to the coherent resonance conjecture, sum and difference resonances are excited by the external driving term $x^{|k|} y^{|\ell|}$ of the frequency $f_{m}$ under the condition

$$
k \nu_{0 x}+\ell \nu_{0 y}=\frac{\kappa_{m}}{1-(1-\eta) C_{m}},
$$

where we have again assumed $\eta_{x}=\eta_{y}(\equiv \eta)$ for simplicity. Equation (15) agrees with Eq. (14) for positive $k$ and $\ell$ when $\nu_{0 x}=\nu_{0 y}\left(\equiv \nu_{0}\right)$, which means that the noncoupling resonances studied in the last subsection have overlapped with the sum resonances of the same orders. Nevertheless, we observed no signature of stop-band splitting in PIC simulations and in S-POD experiment [34]. This supports our assumption that the magnitude of a coherent band shift depends simply on the order number $m$, no matter whether the instability occurs only in a particular direction or in both transverse directions simultaneously.

Compared with the instability in one direction, the $C_{m}$ estimation of coupling resonance is much more troublesome because the emittance growth is very sensitive to the initial emittance condition. Symmetric difference resonances may not always be detectable, for example. The PIC results in Sec. III C and Appendix C have also pointed out the disappearance of a part of the third-order difference resonance bands under the initial condition $I_{k \ell}=0$. Even if we succeeded in activating a difference resonance, the emittance exchange naturally begins leading to the horizontal and vertical density oscillations that can be a serious error source in the evaluation of $C_{m}$. We here focus on sum resonances, expecting that such unwanted effects are hopefully less problematic.

The operating point has to be taken carefully within a region free from the natural resonances under the condition in Eq. (8). As already revealed in Sec. III, many self-fielddriven resonance bands of the second and third orders are running on the $\nu_{0 x}-\nu_{0 y}$ plane. It is necessary to avoid them and preferably even very weak fourth-order bands, so that we can guarantee that the emittance growth observed comes solely from the external-field-driven resonance under the condition (15). After careful test simulations, we decided to put the operating point at $\left(\nu_{0 x}, \nu_{0 y}\right)=(0.142,0.211)$. Although this point is close to the possible third-order sum and fourth-order difference resonance lines, the actual instability bands slightly shift to the higher tune side, which ensures a sufficient distance from these weak natural resonances. As we did in the last subsection, the tune depression $\eta$ and driving perturbation frequency $\kappa_{m}$ are scanned to search for the stop band predicted by Eq. (15).

The coupling resonance of the second order $(m=2)$ is strong and thus easy to find out with relatively weak driving perturbation. Figure 15 clarifies the parameter-dependence of the linear sum resonance excited by the skew quadrupole perturbation $(\propto x y)$. Since $k=\ell=1$ and the operating bare tunes are known, the left-hand side of Eq. (15) takes a certain constant value. We can, therefore, estimate $C_{2}$ from the numerical data in Fig. 15 by fitting Eq. (15) to the peak position of the emittance-growth band. The fitting results were similar in all three types of initial distribution functions as summarized in Table II.

The sextupole $(m=3)$ driving term proportional to $x^{2} y$ was switched on to get the emittance growth chart in 

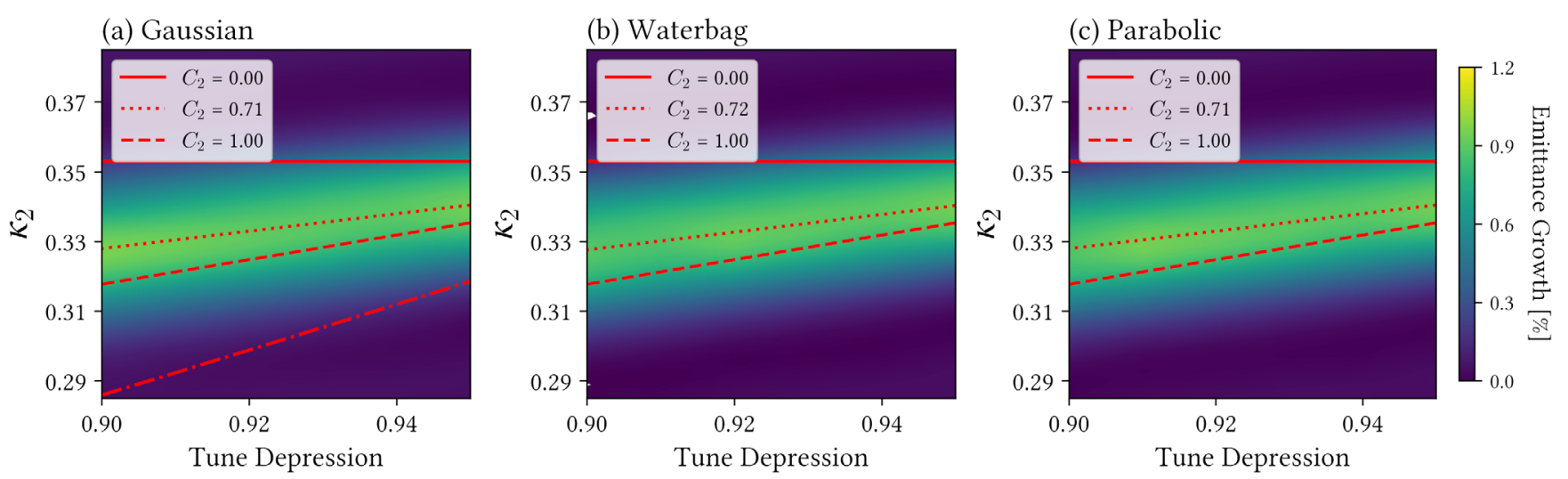

FIG. 15. Emittance growth chart of the sum resonance with $(k, \ell)=(1,1)$ excited by the linear driving perturbation. The perturbation amplitude is set at $0.1 \%$ of the focusing field's in these WARP simulations. The solid and dashed lines correspond to the cases where $C_{2}=0$ (single-particle resonance) and $C_{2}=1$. The dotted line is the location of the stop-band peak fitted by Eq. (15) with $C_{2}=0.71$ for Gaussian and parabolic, and $C_{2}=0.72$ for waterbag. The dash-dotted line in the left panel indicates the incoherent resonance line for a particle with the maximum tune shift.

(a) Gaussian

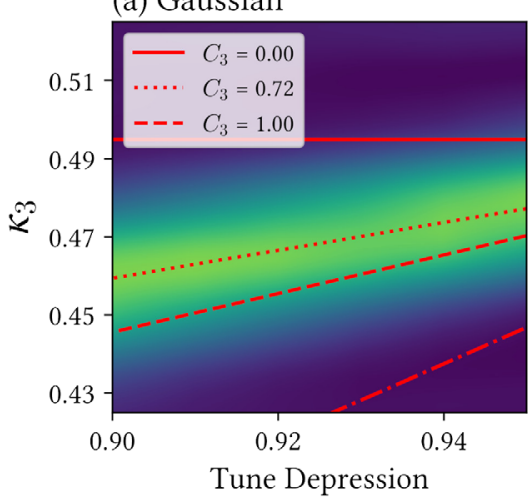

(b) Waterbag

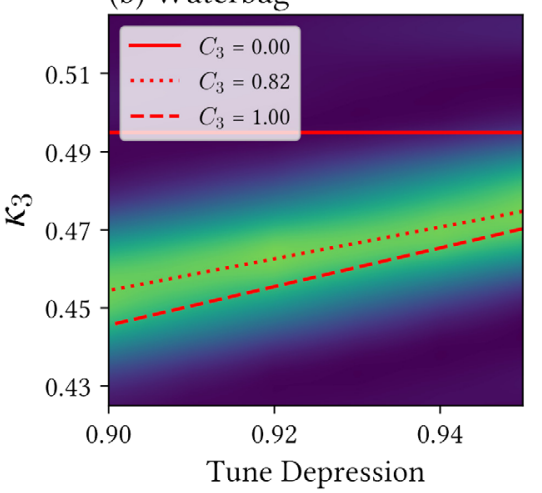

(c) Parabolic

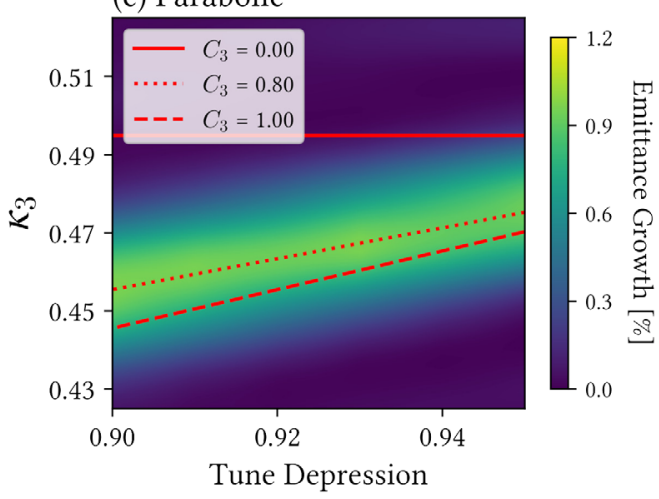

FIG. 16. Emittance growth chart of the sum resonance with $(k, \ell)=(2,1)$ excited by the sextupole driving perturbation. The perturbation amplitude is set at $0.4 \%$ of the focusing field's in these WARP simulations. The dotted line is the location of the stop-band peak fitted by Eq. (15) with $C_{3}=0.72$ for Gaussian, $C_{3}=0.82$ for waterbag, and $C_{3}=0.80$ for parabolic.

Fig. 16. Since the third-order effect is weaker than the second order's, the perturbation amplitude relative to the linear focusing amplitude has been increased from $0.1 \%$ in Fig. 15 to $0.4 \%$ here. The fitting procedure based on the resonance formula in Eq. (15) with $k=2$ and $\ell=1$ leads to $C_{3}=0.72 \pm 0.08$ for the Gaussian distribution, $C_{3}=0.82 \pm 0.03$ for the waterbag distribution, and $C_{3}=0.80 \pm 0.05$ for the parabolic distribution.

The fourth-order effect is even much weaker than the third order's as is evident from the WARP simulation results in Sec. III, which forced us to further raise the perturbation

TABLE II. Coherent tune-shift factors estimated from sum resonance bands excited by skew multipole fields.

\begin{tabular}{lccc}
\hline \hline & $C_{2}$ & $C_{3}$ & $C_{4}$ \\
\hline Gaussian & $0.71 \pm 0.05$ & $0.72 \pm 0.08$ & $\ldots$ \\
Waterbag & $0.72 \pm 0.05$ & $0.82 \pm 0.03$ & $0.84 \pm 0.01$ \\
Parabolic & $0.71 \pm 0.05$ & $0.80 \pm 0.05$ & $0.79 \pm 0.01$ \\
\hline \hline
\end{tabular}

amplitude to $1 \%$ while such a strong external driving force may expedite the growth of the beam tail. The skew octupole field $\left(\propto x^{3} y\right)$ is applied in Fig. 17 to excite the sum resonance with $k=3$ and $\ell=1$. The tune-shift factor evaluated from the PIC data is $C_{4}=0.84 \pm 0.01$ for the waterbag distribution and $C_{4}=0.79 \pm 0.01$ for the parabolic distribution.

In the Gaussian case, we found it difficult to make a reasonable estimate of $C_{4}$. The emittance-growth mountain of the Gaussian beam plotted as a function of $\kappa_{4}$ was not only much wider than the other two cases but also asymmetrically distorted especially when the initial beam density was high. Furthermore, the peak position gradually moves to the higher- $\kappa_{4}$ side as the emittance grows. These effects reduce the estimated value of $C_{4}$ significantly. A similar tendency had been noticed also in the $C_{3}$ evaluation for the Gaussian distribution. We suspect that it is basically the same effect as seen in Fig. 10; namely, the Gaussian tail, even after the $1 \%$ initial truncation, is still working to push 


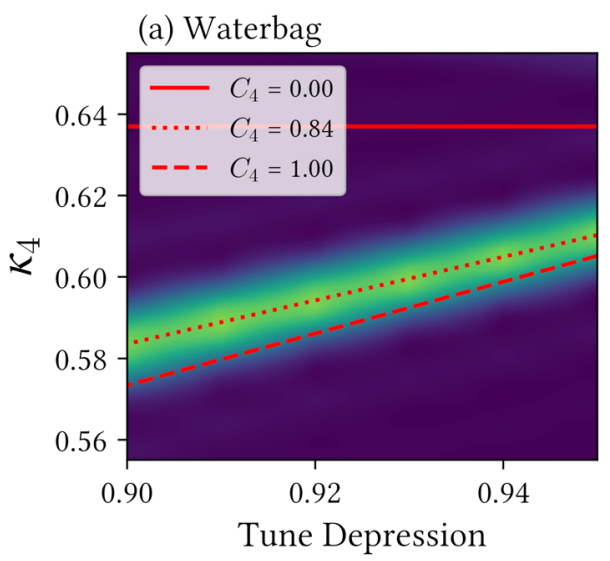

(b) Parabolic

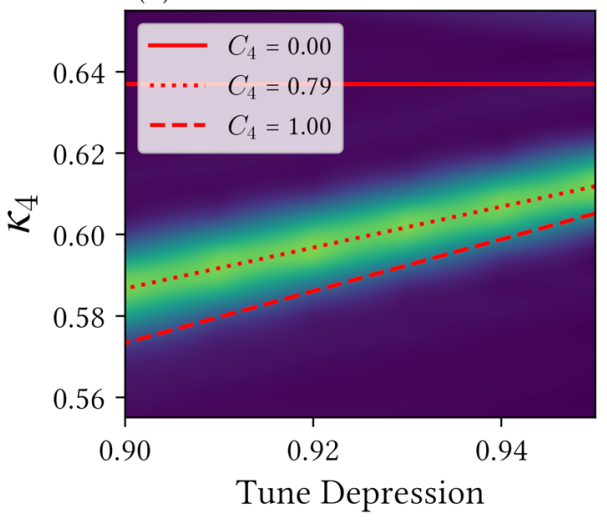

FIG. 17. Emittance growth chart of the sum resonance with $(k, \ell)=(3,1)$ excited by the octupole driving perturbation. The perturbation amplitude assumed in these WARP simulations is $1 \%$ of the focusing field's. The dotted line is the location of the stopband peak fitted by Eq. (15) with $C_{4}=0.84$ for waterbag, and $C_{4}=0.79$ for parabolic. The color bar omitted here is the same as employed in Figs. 15 and 16.

down the estimated $C_{m}$ away from its real value in the beam core.

\section{CONSTRUCTION OF A STABILITY TUNE DIAGRAM}

The operating betatron tunes $\left(\nu_{0 x}, \nu_{0 y}\right)$ of a highintensity ring are decided commonly on the basis of the incoherent picture as illustrated in Fig. 1. We here employ the coherent resonance conjecture in Eq. (8), numerically verified in previous sections, to provide a simple and practically useful guideline for the determination of the optimum machine operating condition. Figure 18 outlines how to find a good operating point $\mathrm{P}$ on the basis of the coherent picture. We no longer rely on the incoherent tune spread and any other model-dependent unobservables. The basic information required for the construction of a stability chart as in Fig. 18 are the rms tune depressions and the lattice design including the distribution of possible loworder nonlinearities. No more information is necessary. Since the beam intensity and emittances at injection are

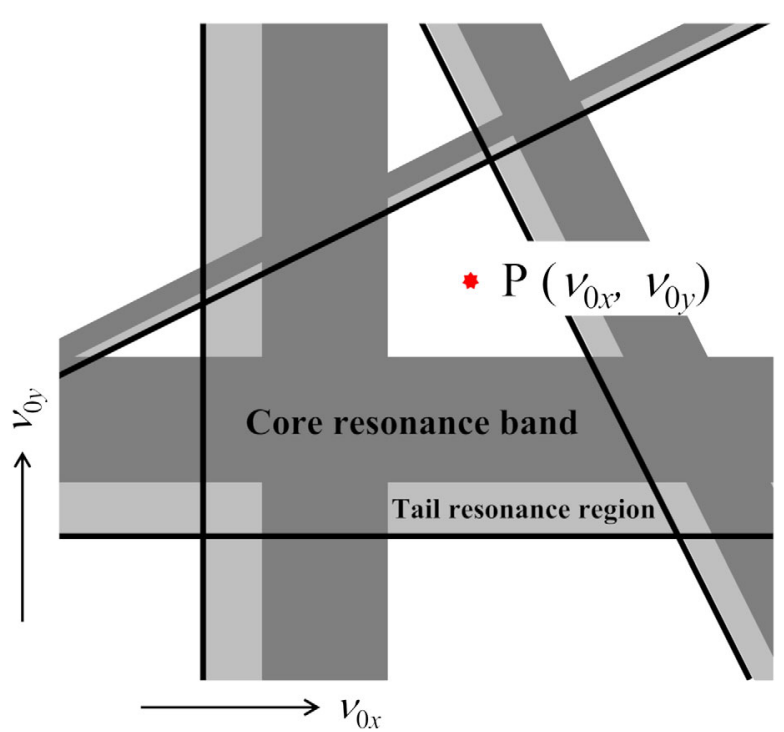

FIG. 18. Determination of a machine operating point on the basis of the coherent concept. The shaded areas represent the coherent core resonance bands whose central positions are predicted by Eq. (8). The width of each instability band generally depends on the resonance strength. We have a possibility of nonnegligible emittance growth also in the areas of a lighter shade where quasi-single-particle resonances can be excited in the beam tail. The horizontal and vertical tail resonance regions adjacent to the half-integer lines may contain a narrow band of the severe dipole $(m=1)$ resonance [39]. The machine operating point $\mathrm{P}$ must be placed within a resonancefree region.

known in any machine, it is straightforward to calculate the tune depressions from the rms envelope equations in Appendix A. The lattice design tells us which driving harmonic numbers can be dangerous. If there exist any known nonlinear potential sources such as sextupole magnets for chromaticity correction, they will enhance the relevant stop bands predicted by Eq. (8) with even $n^{\prime}$.

An important question is what orders of resonances must be taken care of in practice. None of the past Vlasov theories can answer this question, but naturally, we should pay more attention to lower-order resonances rather than higher orders'. Highly nonlinear modes are very weak and thus likely suppressed by the Landau damping mechanism unless strong nonlinear driving forces due to lattice imperfections are present in the machine. As for the self-field-driven resonance, the answer depends on the beam density and on how long the beam stays in the machine.

Our past experience in S-POD experiment indicates that careful consideration to noncoupling coherent resonances of up to the third order $(m \leq 3)$ is always demanded. If the beam goes through a huge number of lattice periods before extraction or only very little emittance growth can be tolerated, we probably need to take care of the next order as well $(m \leq 4)$ [31]. On the other hand, it is uncertain whether we must worry about all coupling resonance lines up to the third order. The importance of each coupling 
resonance band may change depending on initial beam conditions. As demonstrated in S-POD experiment (Appendix B) and PIC simulations (Sec. III), the symmetric coupling potential proportional to $(x y)^{|k|}$ has no effect on a well-conditioned beam with $\varepsilon_{x}=\varepsilon_{y}$ even if the operating point is exactly on the difference resonance line $k \nu_{0 x}-$ $k \nu_{0 y}=0$. The tune diagram in Fig. 6 also shows that a part of the third-order coupling resonance bands are very weakened when the beam is initially in the equipartitioned state. We can even deactivate the emittance exchange mechanism on particular difference resonance lines by imposing the condition $I_{k \ell}=0$ at injection (Appendix C). The situation could be more complex in a real storage ring where the momentum dispersion exists, but in any case, it is recommended to give careful consideration to all second-order and third-order selffield-driven resonances associated with dangerous harmonic numbers. This automatically covers many external-fielddriven resonances of the fourth and sixth orders.

The Fourier analysis of the matched beam envelope will provide us with useful information to judge which harmonic numbers are of practical importance. According to the OY theory, the growth rate and band width of a specific coherent resonance are both proportional to the amplitude of the driving harmonic obtained by Fourier expanding the three-halves power of the space-charge-modified betatron function. If we expand it around the ring, only every $N_{\mathrm{sp}}$ harmonics appear as long as random imperfection fields are negligible. The right-hand side of Eq. (8) is then replaced by $N_{\text {sp }} n^{\prime} / 2$. The lattice design determines which $n^{\prime}$ needs attention. The CERN Proton Synchrotron, for instance, has a ten-fold symmetric structure $\left(N_{\mathrm{sp}}=10\right)$. The matched beam envelope beats fifty times around the ring because each superperiod consists of five identical FDDF blocks. We thus have to be careful especially about the harmonic number $n^{\prime}=5$ with $N_{\text {sp }}=10$ [31].

It is extremely difficult to make a general quantitative argument on the band widths of coherent resonances. They depend not only on the lattice design but also on the particle distribution in phase space. Systematic self-consistent simulations will be required for precise estimation of each band width. Instead of doing time-consuming simulations, we here introduce a simple criterion that enables quick construction of a tune diagram as a starting point for beam stability consideration. In a circular machine, the tune depressions of both transverse directions are not very far from unity. We can put $\eta_{x} \approx \eta_{y}(\equiv \eta)$ in many cases, which permits us to employ the simplified formula in Eq. (9) to determine the central positions of coherent stop bands. The PIC results in Sec. III suggest that any stop bands except for the severe second-orders' are narrower than $\Delta \bar{\nu} / \eta$ that roughly corresponds to the spacing between the two theoretical lines under the conditions $C_{m}=0$ and $C_{m}=1$ in Eq. (9). We, therefore, assume the widths of all stop bands to be equal to $\Delta \bar{\nu} / \eta$. Since the nonlinear bands' widths are probably overestimated, this criterion gives us a sufficient safety margin. When $\varepsilon_{x} \approx \varepsilon_{y}\left(\equiv \varepsilon_{\perp}\right)$ and $\Delta \bar{\nu} / \nu_{0} \ll 1, \Delta \bar{\nu} / \eta$ can be evaluated from

$$
\frac{\Delta \bar{\nu}}{\eta} \approx \frac{\lambda R r_{\mathrm{p}}}{4 \varepsilon_{\perp} \beta^{2} \gamma^{3}},
$$

where $\beta$ and $\gamma$ are the Lorentz factors, $\lambda$ is the line density of the beam, $R$ is the average radius of the ring, and $r_{\mathrm{p}}$ is the classical particle radius. The size of $\Delta \bar{\nu} / \eta$ is much smaller than the incoherent tune spread of a Gaussian beam (see Appendix A).

There is a narrow gap in-between a coherent instability band of the width $\Delta \bar{\nu} / \eta$ and the adjacent zero-intensity $(\eta=1)$ resonance line. The gap area, i.e., the tail resonance region indicated with a lighter shade in Fig. 18, is potentially dangerous because of the following reasons. First, theoretically, the emittance growth is more severe near the low-tune boundary of a coherent stop band (see the sawtooth profile of the waterbag and parabolic stop bands in Fig. 9). Each stop band moves toward the zero-intensity line whenever the beam density is lower than the design value or lowered by any unexpected instability. Second, the betatron amplitudes of the tail particles that have small incoherent tune shifts can grow resulting in beam loss. The tail resonance region becomes less troublesome if we somehow form and inject a well-matched beam with a sharp edge. The waterbag and parabolic beams actually have a resonance-free gap above the zero-intensity resonance line as can be seen from the examples in Fig. 9. In reality, however, any beam will more or less have a tail around the core. We thus better avoid the region in-between a single-particle resonance line and its neighboring coherent band to prevent tail particles from resonating at their incoherent tunes.

The horizontal and vertical quadrupole resonances $(m=2)$ at every half-integer tune per lattice period are accompanied by a strong dipole $(m=1)$ resonance [39]. Since the dipole stop band is very narrow and ideally has no space-charge-induced shift $\left(C_{1}=0\right)$, it is always located close to half-integer lines, $\nu_{0 x(0 y)}=N_{\text {sp }} / 2, N_{\text {sp }}, 3 N_{\text {sp }} / 2, \ldots$. The seed of the coherent dipole resonance is a finite deviation of the beam centroid from the design closed orbit. Unlike other collective modes $(m \geq 2)$, the dipole mode can be stabilized in principle by properly correcting the position of the beam centroid.

Let us try to draw a stability tune diagram for a particular machine, applying the general rule described above. As an example, we consider the lattice of the rapid cycling synchrotron (RCS) at the Japan Proton Accelerator Research Complex (J-PARC) [63]. So far, the spacecharge-induced resonance in the RCS has been discussed on the basis of the incoherent tune spread as in Fig. 1 [64]. The RCS, whose circumference is $348.333 \mathrm{~m}$, has a threefold symmetric structure $\left(N_{\mathrm{sp}}=3\right)$. The injection painting scheme has been employed to control the density profile of a high-power proton beam for space-charge mitigation (while forming an approximately uniform particle density 
to reduce the incoherent tune spread is not necessarily beneficial because what really matters is the resultant band widths of coherent resonances rather than the size of the tune spread). After the ideal painting at the kinetic energy of $400 \mathrm{MeV}$ to form an approximately uniform bunch, the unnormalized edge emittance reaches $200 \pi \mathrm{mm} \cdot \mathrm{mrad}$ in both transverse directions. This corresponds to $\varepsilon_{\perp} \approx$ $50 \mathrm{~mm} \cdot \mathrm{mrad}$. The number of protons contained in a single bunch of $92 \mathrm{~m}$ long is about $4.165 \times 10^{13}$. Substituting these numbers into Eq. (16), we obtain $\Delta \bar{\nu} / \eta \approx 0.13$. The rms tune depression is 0.98 in the operating region around $\nu_{0} \approx 6.5$.

We here take all possible self-field-driven resonance bands of the second and third orders into account. The numerical study in the last section has concluded that the coherent tune-shift factor is in the range $0.7 \lesssim C_{m} \lesssim 0.9$ for low-order collective modes. Recalling the fitting results in Tables I and II, we assume that $C_{2}=0.75$ and $C_{3}=0.80$. The essential feature of the stability diagram does not change much even if slightly different $C_{m}$ factors are adopted. The resultant distribution of the core resonance bands plus the neighboring tail resonance regions are sketched in Fig. 19. The width of the difference resonance band along $\nu_{0 x}-$ $\nu_{0 y}=0$ has been disregarded because $\varepsilon_{x} \approx \varepsilon_{y}\left(I_{1,-1} \approx 0\right)$ in the RCS. It is, however, not recommended to put the operating point too close to this line unless the emittance equality is guaranteed. The coupling resonance is only deactivated under the condition $I_{1,-1} \approx 0$ but still there. The emittance exchange mechanism will immediately become active once the above condition is broken somehow.

It turns out from the diagram that the resonance-free area is quite limited in the RCS due to the low lattice symmetry. The coherent instability in a few stop bands indicated with the darkest shade can be enhanced by quadrupole and sextupole magnets. Three families of sextupole magnets have actually been installed in the RCS for chromaticity correction. These external-field-driven stop bands have overlapped with the self-field-driven stop bands of the same orders. The self-field-driven instabilities above $\nu_{0 x(0 y)}=$ 6.75 are caused by the linear mode $(m=2)$ and thus particularly dangerous at high beam density. Figure 19 reveals several operating regions free from low-order resonances. The widest one is located just below the bare tunes of 6.5. After a careful tune survey, the operating point of the RCS has been chosen at around $\left(\nu_{0 x}, \nu_{0 y}\right)=$ $(6.45,6.42)$ [63], which is consistent with the theoretical prediction in Fig. 19.

If we add the fourth-order bands, the resonance-free regions shrink even more. The above operating point is then covered by the possible octupole instability bands corresponding to $\left(k, \ell, n^{\prime}\right)=(4,0,3 \times 17)$ and $(0,4,3 \times 17)$ in Eq. (9). The RCS performance may, therefore, be improved somewhat by moving the current operating point slightly below the lines $\nu_{0 x(0 y)}=6.375$. The present discussion has focused on especially important resonances associated with

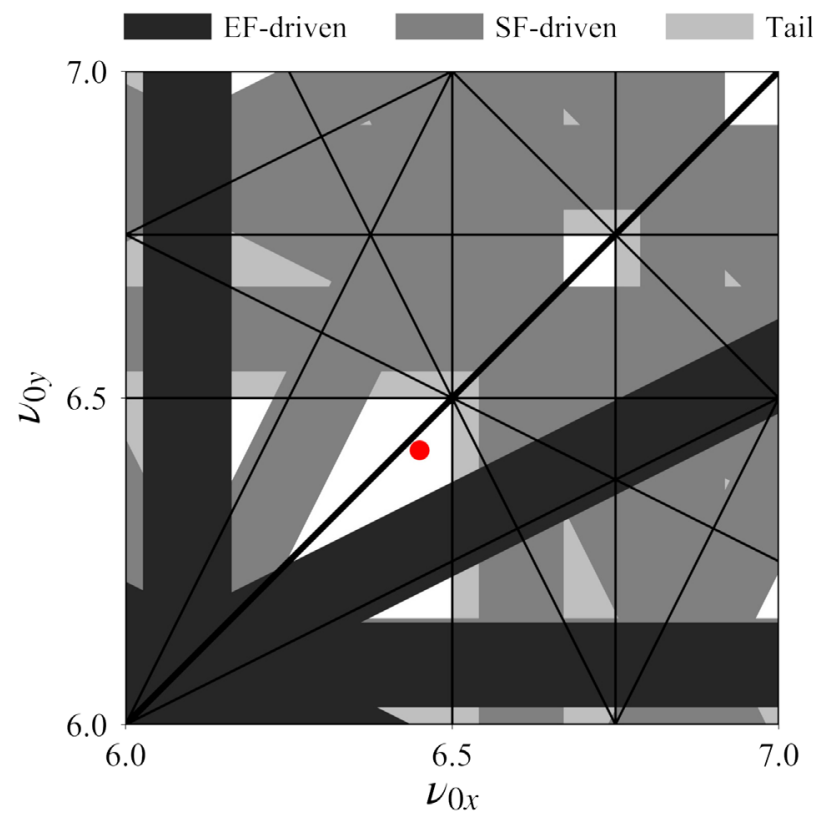

FIG. 19. Stability tune diagram based on the fundamental parameters of the RCS at J-PARC. The three different types of instability bands, produced by the self-field-driven, externalfield-driven, and tail-induced resonances, are plotted up to the third order. The coherent resonance condition in Eq. (9) has been used together with the tune-shift factors $C_{2}=0.75$ and $C_{3}=0.80$. The rms tune depression is fixed at 0.98 , considering the beam parameters after the transverse injection painting at the RCS. For the sake of simplicity, all stop bands are assumed to have an identical width except for the symmetric difference resonance along $\nu_{0 x}-\nu_{0 y}=0$ (thick solid line) where the coupling effect should be less dangerous under the operating condition of the RCS. The thin solid lines show the positions of the resonances at zero-intensity limit $(\eta \rightarrow 1)$. A red dot on the picture indicates the typical operating point of the RCS, i.e., $\left(\nu_{0 x}, \nu_{0 y}\right)=(6.45,6.42)[63]$.

the harmonic numbers of integral multiples of the lattice superperiodicity $N_{\mathrm{sp}}$. If the machine contains any sources of strong imperfections that significantly affect the original lattice symmetry, we have to take more resonance bands into account. As emphasized repeatedly, the error fields only excite the resonances with even $n^{\prime}$ in Eq. (9).

\section{CONCLUDING REMARKS}

Self-consistent PIC simulations have been performed systematically to corroborate a conjecture on the 2D betatron resonance in high-intensity rings where the space-charge potential plays an important role. The proposed resonance formula in Eq. (8) is model-independent, free from any incoherent quantities and based only on a few observable parameters. We have confirmed that Eq. (8), despite its remarkable simplicity, works to reproduce the basic features of numerically observed resonance stop bands in the tune diagram. At high beam density, the factor $1 / 2$ on 
the right-hand side can give rise to a lot of extra instability bands overlooked or underestimated in previous resonance studies. No clear indication of simultaneous existence of the coherent and incoherent resonance mechanisms has been identified in a beam core (while the quasi-single-particle resonance in the beam tail around the core can cause rms emittance growth and resultant particle losses).

Any circular machine intended to store an intense hadron beam for a long period should be designed to have as high lattice symmetry as possible, so that the number of selffield-driven instability bands is minimized. The coherent tune-shift factor is found to be always smaller than unity for any low-order collective oscillation modes. The present study suggests that the locations of practically important stop bands can be estimated with the tune-shift factor that depends only on the resonance order $m$; we can assume $C_{m}$ to be constant over the whole tune space independently of initial beam conditions, at least, in the beam-density range achievable in storage rings.

On the basis of the coherent resonance concept wellestablished through extensive theoretical effort in the past [10-12,19-30], we have proposed a new prescription for constructing the stability tune diagram without the use of the incoherent tune spread. Although any real machines contain diverse artificial sources of transverse perturbing fields which complicate the stop-band distribution on the diagram, the simple rule described in Sec. V is helpful to spot preferable operating regions very quickly as the first step toward the best machine performance. Considering long CPU time necessary for high-precision PIC simulations of long-term beam stability, it is certainly meaningful to know the locations of possible intrinsic stop bands in advance. The accurate information of external fields (e.g., the arrangement of nonlinear magnets, possible sources of strong error fields, etc.) peculiar to an individual machine will improve the reliability of the stability chart.

Many recent numerical studies on space-charge issues have assumed Gaussian beams. Taking the Gaussian model sounds reasonable but there is no guarantee that any real beams in high-intensity circular machines have the exact Gaussian distribution in phase space. The details of an initial beam configuration strongly depend on how the beam was injected and accumulated in a ring. The highpower proton beam provided by the injection painting scheme at the RCS actually has a phase-space profile rather different from the Gaussian configuration. The long Gaussian tail also brings about the side effects that obscure the core dynamics. It is thus dangerous to look only at Gaussian-based predictions. To be on the safe side, we should care about what is expected in other types of reasonably realistic beam core as well. Furthermore, even after very careful injection, the initial beam distribution is not so close to the perfect stationary state precisely matched to the lattice and, as a result, includes a complex distortion pattern in phase space. Such imperfection can be an unfavorable seed of coherent instability as shown in Appendix D. The core of a real beam is probably more susceptible to coherent nonlinear resonances than the wellmatched core employed in computer simulations.

The conventional resonance conditions in Sec. I, commonly used to plot the tune diagram, have not taken the synchrotron motion explicitly into account. Similarly, the possibility of synchro-betatron coupling has been disregarded here. The present empirical rule is restricted to the transverse dynamics. Much further investigation is required to establish an analogous rule for intense bunched beams.

\section{ACKNOWLEDGMENTS}

This work is supported in part by Japan Society for the Promotion of Sciences (JSPS) KAKENHI Grant No. 18H03472. The authors would like to thank K. Moriya for providing the S-POD data on the coupling resonance discussed in Appendix B. They also express their sincere gratitude to $\mathrm{S}$. Machida for valuable discussion on resonance issues in high-intensity rings and to $\mathrm{H}$. Hotchi and $\mathrm{H}$. Harada for useful information about the rapid cycling synchrotron at J-PARC. One of the authors (K. K.) is indebted to K. Fukushima for his kind assistance in running the WARP code.

\section{APPENDIX A: THE RMS TUNE SHIFT AND INCOHERENT TUNE SPREAD}

The horizontal and vertical rms beam sizes $(a, b)$ satisfy

$$
\begin{aligned}
& \frac{d^{2} a}{d s^{2}}+K_{x}(s) a-\frac{\varepsilon_{x}^{2}}{a^{3}}-\frac{K_{\mathrm{sc}}}{2(a+b)}=0, \\
& \frac{d^{2} b}{d s^{2}}+K_{y}(s) b-\frac{\varepsilon_{y}^{2}}{b^{3}}-\frac{K_{\mathrm{sc}}}{2(a+b)}=0,
\end{aligned}
$$

where $\varepsilon_{x(y)}$ is the horizontal(vertical) rms emittance, $K_{x(y)}$ is the horizontal(vertical) focusing function determined by the AG lattice structure, $K_{\mathrm{sc}}$ is the perveance proportional to the beam's line density, and $s$ is the path length along the reference orbit [50]. Sacherer proved that Eqs. (A1) hold for any distribution function with elliptical symmetry in the transverse real space. The rms tune shifts in the horizontal and vertical directions are evaluated by substituting the stationary solutions of Eqs. (A1) into

$\Delta \bar{\nu}_{x}=\nu_{0 x}-\frac{\varepsilon_{x}}{2 \pi} \int_{s}^{s+L} \frac{d s}{a^{2}}, \quad \Delta \bar{\nu}_{y}=\nu_{0 y}-\frac{\varepsilon_{y}}{2 \pi} \int_{s}^{s+L} \frac{d s}{b^{2}}$,

where $L$ is the ring circumference (or the length of single lattice period). $a$ and $b$ in Eq. (A2) represent the matched beam envelopes with space charge, both of which have the periodicity of $L$. The rms tune depressions are defined by $\eta_{x}=\left(\nu_{0 x}-\Delta \bar{\nu}_{x}\right) / \nu_{0 x}$ and $\eta_{y}=\left(\nu_{0 y}-\Delta \bar{\nu}_{y}\right) / \nu_{0 y}$. 

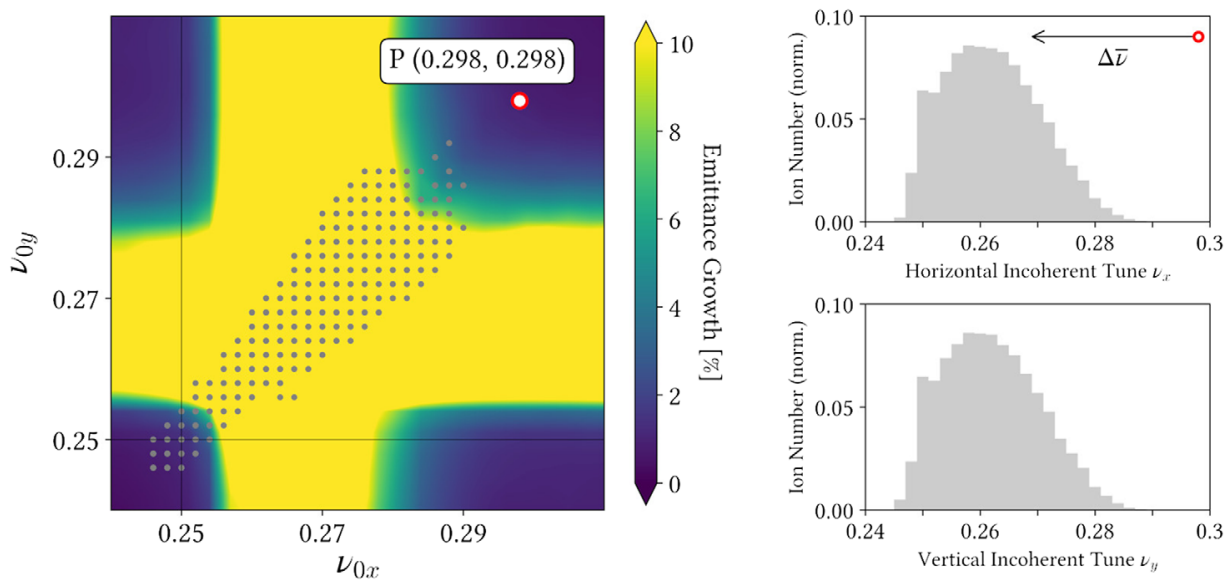

FIG. 20. Example of the incoherent tune spread of a Gaussian beam with $\eta=0.9$. The emittance growth is evaluated from WARP simulations that have assumed the same numerical conditions as in Fig. 3 except for the transport distance extended to 200 AG periods here. Gray dots represent the approximate incoherent tunes of individual particles when the operating point is set at $\left(\nu_{0 x}, \nu_{0 y}\right)=$ $(0.298,0.298)$ where the matched Gaussian beam is completely stable. The right panels show the particle-number histograms plotted as a function of the incoherent tunes $\nu_{x}$ and $\nu_{y}$. The ordinate (the number of particles) has been normalized with the total number of macroparticles used for the PIC simulation. The arrow in the upper right panel indicates the size of the rms tune shift.

For comparison, let us consider the incoherent tune shift in the Gaussian model that has been frequently employed in past simulation studies and to plot an incoherent tune spread area in the tune diagram. Assuming the exact Gaussian density profile to be maintained (no Debye screening), we can make a crude estimate of the maximum incoherent tune shifts in the horizontal and vertical directions from

$$
\begin{aligned}
\max \left(\Delta \nu_{x}^{\text {Gauss }}\right) & \approx \frac{\lambda r_{\mathrm{p}} R^{2}}{\nu_{0 x} \beta^{2} \gamma^{3}} \frac{1}{\bar{a}(\bar{a}+\bar{b})}, \\
\max \left(\Delta \nu_{y}^{\text {Gauss }}\right) & \approx \frac{\lambda r_{\mathrm{p}} R^{2}}{\nu_{0 y} \beta^{2} \gamma^{3}} \frac{1}{\bar{b}(\bar{a}+\bar{b})},
\end{aligned}
$$

where $\bar{a}$ and $\bar{b}$ denote the average rms beam sizes [65]. These formulas are not self-consistent but could be used as an approximate measure that helps us to see the size of a typical incoherent tune spread. It is possible to show, by using the smooth approximation, that $\max \left(\Delta \nu_{x(y)}^{\mathrm{Gauss}}\right) \approx\left(1-\eta_{x(y)}^{2}\right) \nu_{0 x(0 y)}$. Since $\Delta \bar{\nu}_{x(y)}=\left(1-\eta_{x(y)}\right) \nu_{0 x(0 y)}$, the maximum incoherent tune shift in a Gaussian beam is roughly twice as large as the model-independent rms tune shift at low density.

The left panel in Fig. 20 is the tune diagram near the operating region where the severe horizontal and vertical resonance bands of the quadrupole mode intersect. The emittance growth after beam transport over 200 sinusoidal focusing cells is evaluated at many different operating points through WARP simulations starting from initially matched Gaussian beams. The tune depressions are fixed to 0.9 in both transverse directions everywhere. Gray dots on the picture indicate the average positions of individual macroparticles when the operating point is set at $\left(\nu_{0 x}, \nu_{0 y}\right)=$ $(0.298,0.298)$ where no emittance growth was detected, at least, over $5000 \mathrm{AG}$ periods. The area of the incoherent tune spread is obviously wider than the value of $\Delta \bar{\nu}=$ $(1-\eta) \nu_{0}=0.0298$ as well as the observed instability bands.

Particularly noteworthy is the fact that a lot of particles deep inside the core (thus with large incoherent tune shifts) have satisfied the incoherent resonance conditions $4 \nu_{x(y)} \approx 1$. Nevertheless, the beam is completely stable, which supports our conclusion that no serious resonance is activated in the matched core under the incoherent condition in Eq. (3). If we trust the conventional rule in Fig. 1, the operating point $\mathrm{P}$ has to be put further away from the single-particle resonance lines $4 \nu_{0 x(0 y)}=1$ to prevent possible beam loss, but that is totally unnecessary. We found no instability at this operating point even when a rather strong fourth-order error field (1\% of the focusing strength) was added to the external potential.

It is instructive to refer to the case of higher beam density. The tune depression is lowered from 0.9 to 0.8 in Fig. 21. The operating point has been moved to $\left(\nu_{0 x}, \nu_{0 y}\right)=$ $(0.353,0.353)$ to avoid the coherent quadrupole resonance bands wider than those in Fig. 20. Although this operating point is above the third-order single-particle resonance lines $\nu_{0 x(0 y)}=1 / 3$, it has not reached the edge of the coherent sextupole resonance bands shifted to the higher tune side. The beam should, therefore, be free from serious core instability. The incoherent tunes (gray dots) in Fig. 21 are evaluated by Fourier-analyzing the single-particle trajectories near the end of a long transport channel. The tune-spread area has become much wider than the $\eta=0.9$ case in Fig. 20. Many core particles with large incoherent tune shifts are found again around the quarter-integer lines $\nu_{0 x(0 y)}=1 / 4$, but the beam core was stable even after the inclusion of a fourth-order imperfection field. On the other hand, we notice that a small number of particles are 


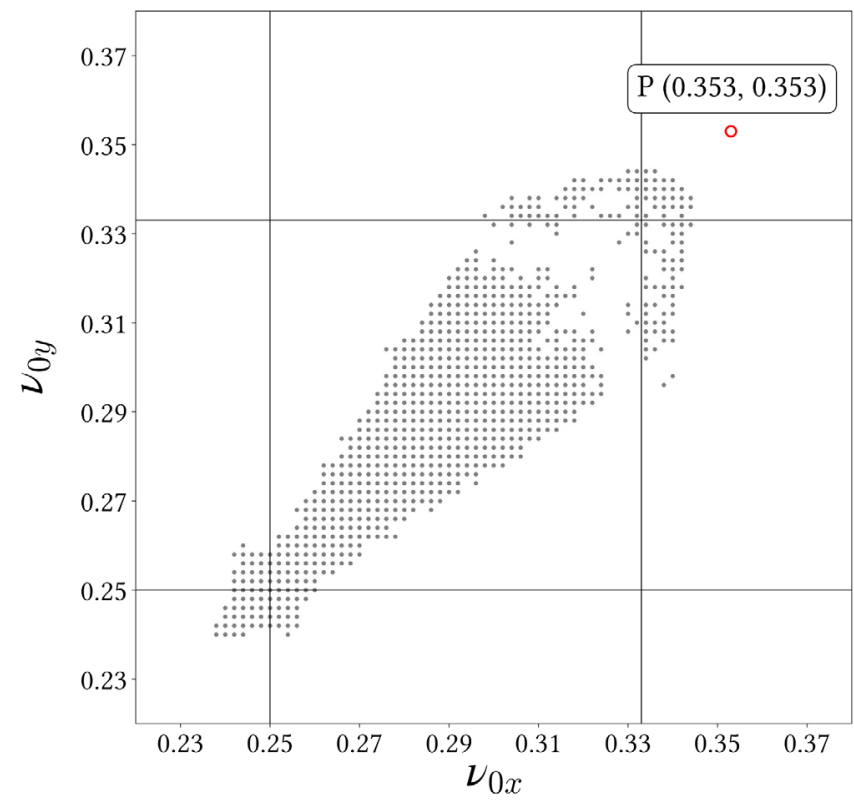

FIG. 21. Example of the incoherent tune spread of a Gaussian beam with $\eta=0.8$ ( $2 \mathrm{D}$ WARP result). The beam transport distance is extended to $1000 \mathrm{AG}$ periods (see Fig. 22). The operating point is set at $\left(\nu_{0 x}, \nu_{0 y}\right)=(0.353,0.353)$. The singleparticle trajectories near the end of the transport channel are Fourier analyzed to figure out the incoherent tune spread. The single-particle resonance lines of the third and fourth orders, i.e., $\nu_{0 x(0 y)}=1 / 3$ and $\nu_{0 x(0 y)}=1 / 4$, are drawn for reference.

distributed along $\nu_{0 x(0 y)}=1 / 3$. They are the tail particles captured by the quasi-single-particle resonance with the oscillating core potential. The time evolution of the rms emittance growth is plotted in Fig. 22 together with the beam profile in real space. An initial emittance jump by about $2 \%$ is attributed to a weak mismatch and has nothing to do with any core instability. The gradual increase of the $100 \%$ emittance is caused largely by the tail particles near $\nu_{0 x(0 y)}=1 / 3$ in Fig. 21. The emittance growth (except for the initial jump) can be made much smaller by disregarding the contribution from just one percent of particles in the tail. The right panels in Fig. 22 show the real-space profiles of the beam before and after the one-percent removal of the tail at the exit of the 1000th cell.

\section{APPENDIX B: EXPERIMENTAL DATA FROM S-POD}

The collective motion of an ion plasma confined in a linear Paul trap (LPT) is almost equivalent to that of a charged particle beam in an AG transport channel. The S-POD, designed on the basis of this fact, offers systematic experimental data to support numerical and theoretical studies of beam dynamics. Figure 23 is an experimentally obtained tune diagram consisting of over four thousand data points from ion-loss measurements performed independently with different combinations of $\left(\nu_{0 x}, \nu_{0 y}\right)$ [31]. Similarly to the PIC simulations in Secs. III and IV, the sinusoidal focusing potential is used for transverse ion confinement. The picture reveals the existence of six clear instability bands running horizontally and vertically. In addition to these resonance lines, a few weak coupling resonance bands are recognizable. Figure 24 clarifies the dependence of ion losses on initial plasma density. The measurements have been done along the line $\nu_{0 x}=$ $\nu_{0 y}\left(\equiv \nu_{0}\right)$ on which the three horizontal and three vertical instability bands in Fig. 23 intersect near $\nu_{0}=1 / 6,1 / 4$, and $1 / 3$. In each measurement, the ion plasma is stored in the LPT for $10 \mathrm{~ms}$ corresponding to the transport distance over $10^{4}$ AG periods.

When the initial plasma density is low, ion losses are detected only at $\nu_{0} \approx 1 / 3$ (see the bottom panel in Fig. 24). Considering the weakness of the self-field potential in the low-density regime, the external-field-driven resonance with even $n^{\prime}$ must be playing a dominant role there. Theoretically, the lowest-order mode that can be unstable at $\nu_{0} \approx 1 / 3$ is the sextupole $(m=3)$. The primary cause
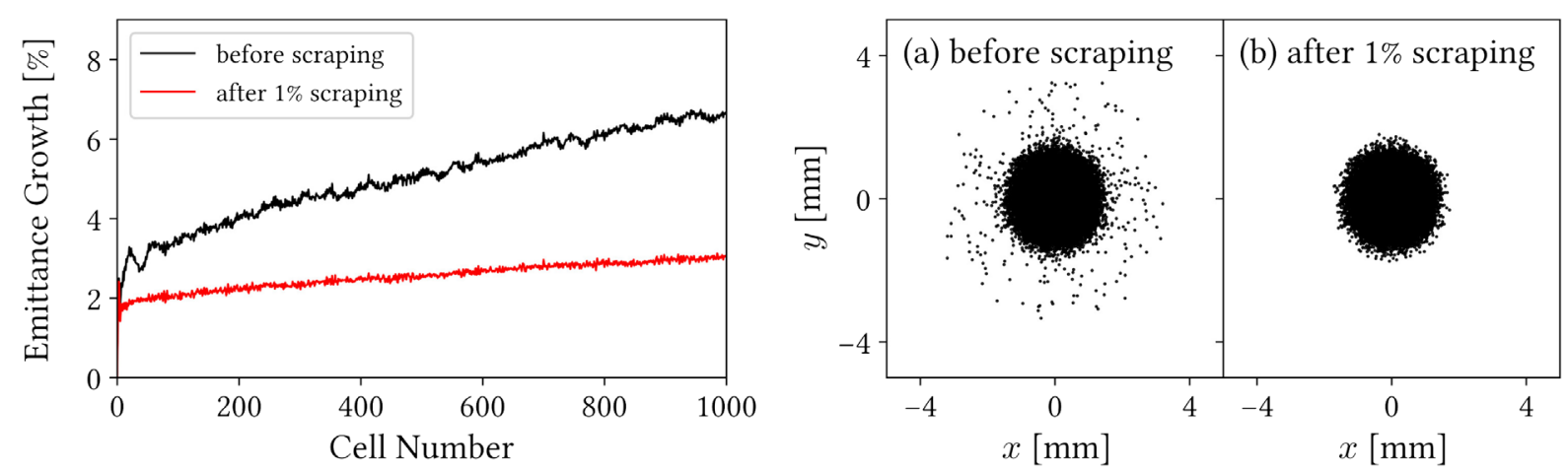

FIG. 22. Time evolution of the emittance growth of the Gaussian beam assumed in Fig. 21. The black solid line is the growth calculated from the whole beam (100\% rms emittance). The red line is based on the truncated distribution in which one percent of tail particles are disregarded ( $99 \%$ rms emittance). A sudden jump of the emittance growth $(\sim 2 \%)$, which occurs within the first few AG cells, is due to the self-organization of the phase-space distribution triggered by a weak initial mismatch to the lattice. The final crosssectional views of the beam before and after the $1 \%$ tail scraping are exhibited in the right panels. 


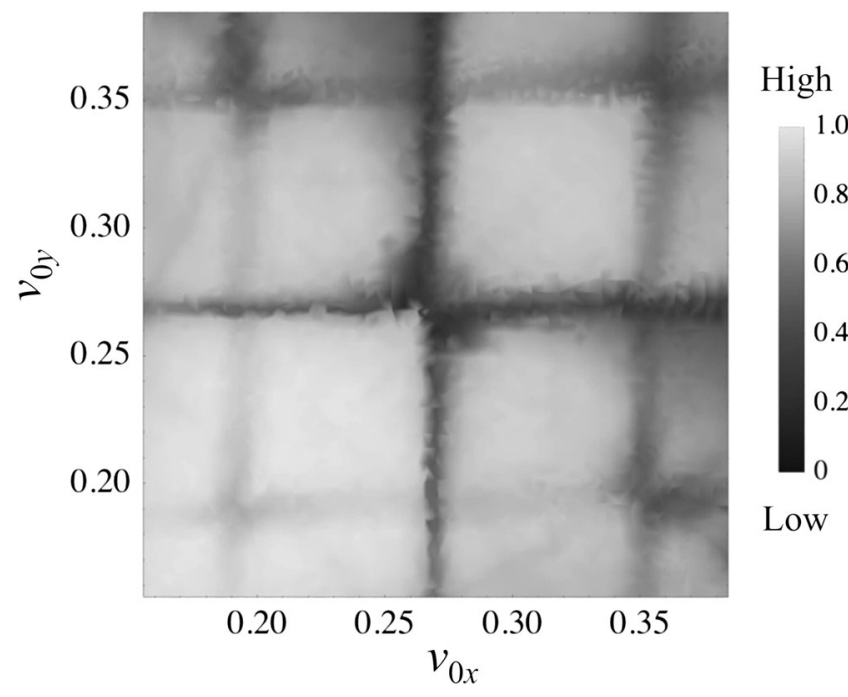

FIG. 23. Tune diagram experimentally obtained with S-POD [31]. The number of ions remaining in the LPT after $10^{4} \mathrm{AG}$ periods is measured at 4450 different operating points. The initial ion number $N_{\text {ion }}$ is fixed at about $10^{7}$ in this example. The shading of gray scale represents the rate of surviving ions. The ion-loss rate is higher in the area of a darker shade.

of the narrow instability band observed in Fig. 24(c) is, therefore, the third-order imperfection field due to mechanical errors in the LPT structure. Note that higherorder error fields have no discernible effect at low density within the present timescale.

The other two instabilities at $\nu_{0} \approx 1 / 6$ and $1 / 4$ are manifested gradually as we increase the initial number of ions in the LPT. Ion losses at $\nu_{0} \approx 1 / 4$ are particularly severe which eventually surpass those at the third-order stop band above $\nu_{0}=1 / 3$ (see the top panel in Fig. 24). The instability at $\nu_{0} \approx 1 / 6$ is also more enhanced with increasing plasma intensity. These observations lead us to a conclusion that the resonances at $\nu_{0} \approx 1 / 6$ and $1 / 4$ are driven mainly by the natural self-field potential. The shifts of the three instability regions toward the higher tune side are due to the effect of $\Delta \bar{\nu}$ in the resonance condition. A question is what order of resonance has caused these ion losses.

It is most natural to seek the origin of observed ion losses in the resonance of as low an order as possible. Accepting the conjecture in Eq. (8), we realize that only the linear $(m=2)$ and first nonlinear $(m=3)$ resonances are sufficient to explain the results in Figs. 23 and 24. Let us temporarily assume that the resonance at $\nu_{0} \approx 1 / 6$ is driven by the sixth-order error field. Then we find it difficult to explain why it completely disappears at low density. The resonances of the lower (fourth and fifth) orders are also nearly invisible in Fig. 24(c). Even if we suppose that this resonance is the six-order self-field-driven instead of external-field-driven, we still have trouble explaining the absence of other lower-order resonance bands in Fig. 23 at high density. The most straightforward answer to the question above is thus as follows: the instability of the (a) $N_{\text {ion }} \approx 10^{7}$

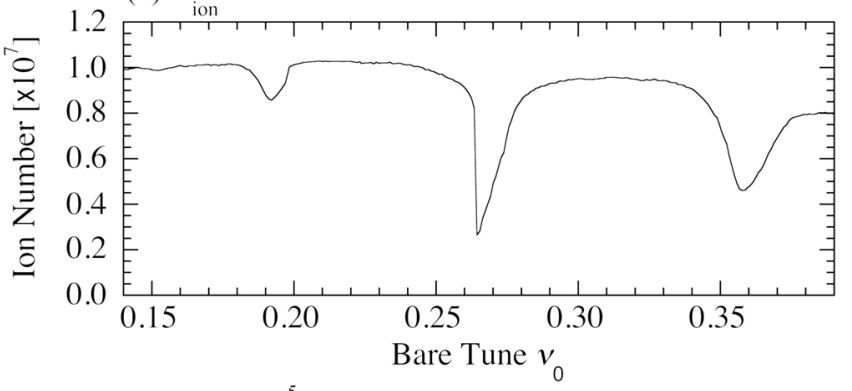

(b) $N_{\text {ion }} \approx 10^{5}$

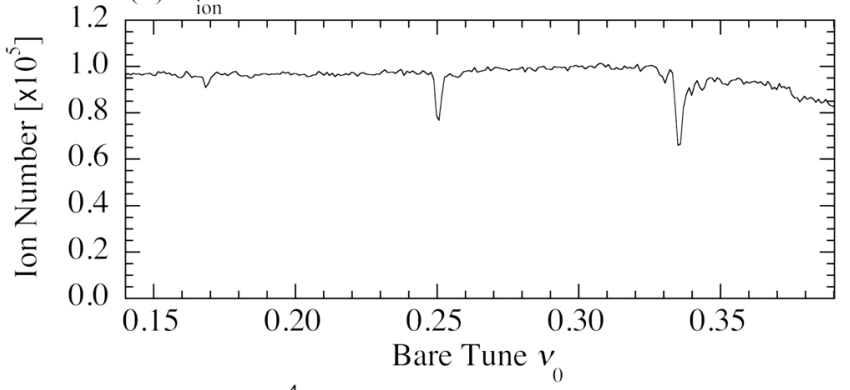

(c) $N_{\text {ion }} \approx 10^{4}$

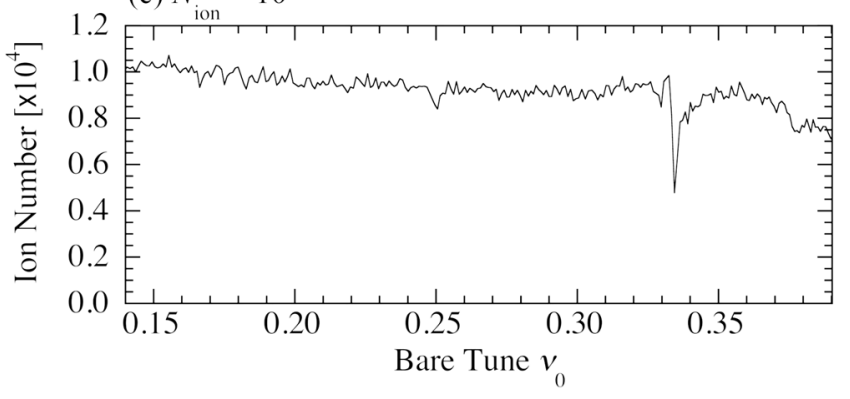

FIG. 24. Dependence of the three major stop bands on plasma intensity. The number of ions remaining in the LPT after $10^{4} \mathrm{AG}$ periods is measured in S-POD at over 200 different operating points. The horizontal and vertical bare tunes are always equalized, i.e., $\nu_{0 x}=\nu_{0 y}\left(\equiv \nu_{0}\right)$, which means that the experiment has been performed along the straight line $\nu_{0 x}-\nu_{0 y}=0$ in the tune diagram. The initial ion number $N_{\text {ion }}$ in the LPT is decreased from the top panel to the lower: (a) $N_{\text {ion }} \approx 10^{7}$, (b) $N_{\text {ion }} \approx 10^{5}$, and (c) $N_{\text {ion }} \approx 10^{4}$.

linear $(m=2)$ mode is responsible for the most serious ion losses at $\nu_{0} \approx 1 / 4$ while the sextupole $(m=3)$ mode for other two resonance bands at $\nu_{0} \approx 1 / 6$ and $1 / 3$. A similar argument applies to another self-field-driven resonance band that appears at $\nu_{0} \approx 1 / 8$ when the plasma storage period is considerably extended [31]. The instability of the octupole $(m=4)$ mode should be the main cause, rather than of the eighth $(m=8)$ or higher orders'.

The possible coupling resonance band along $\nu_{0 x}-\nu_{0 y}=0$ is undetectable in Fig. 23 just like the PIC simulation results in Sec. III. The ion-loss measurements in Fig. 24 have been conducted along this line, but no noticeable instability can be found except in the regions of the three noncoupling resonances discussed above. In these experiments, we used isotropic ion plasmas produced under the 

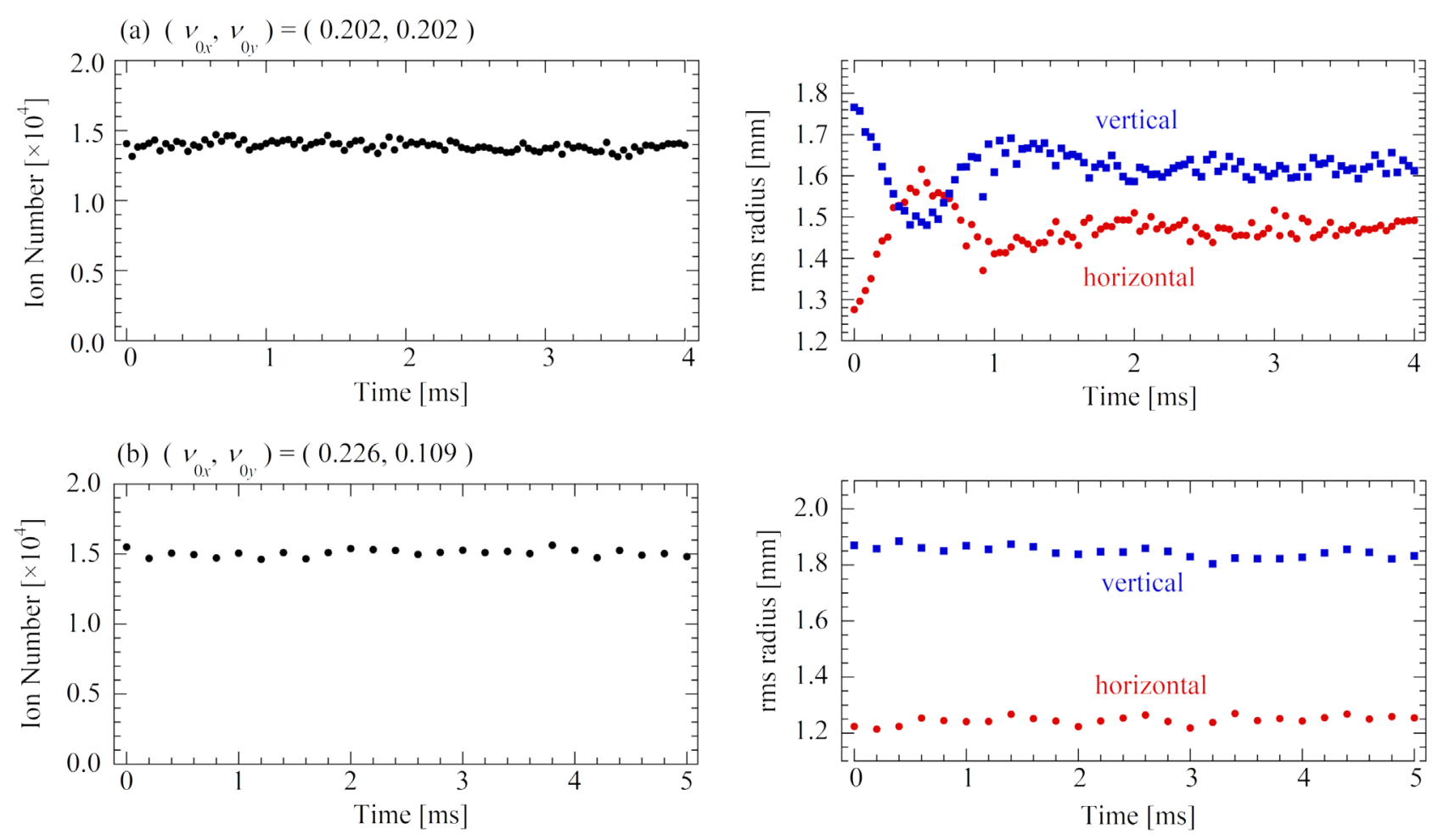

FIG. 25. Time evolution of the ion number (left) and transverse plasma extent (right) measured in S-POD. The ion plasma used for this experiment is initially anisotropic. The operating point of the LPT is adjusted to: (a) $\left(\nu_{0 x}, \nu_{0 y}\right)=(0.202,0.202)$, and (b) $\left(\nu_{0 x}, \nu_{0 y}\right)=(0.226,0.109)$.

condition $\nu_{0 x}=\nu_{0 y}$. Any driving potential symmetric with respect to $x$ and $y$ is then unable to activate the difference resonance on $\nu_{0 x}-\nu_{0 y}=0$. An anisotropic plasma with unequal emittances $\left(\varepsilon_{x} \neq \varepsilon_{y}\right)$ is necessary to verify the existence of this coupling resonance.

There are several ways to provide an anisotropic plasma in the LPT. We employed the dipole electric field generated by applying low pulse voltages of opposite signs to the horizontal pair of the quadrupole rods. An ion bunch is kicked several times by the pulsed dipole field and partially scraped with the electrodes, which develops an imbalance between the horizontal and vertical initial emittances. The timing and strengths of the dipole kicks are designed such that the centroid of the plasma comes back to the LPT axis after the horizontal scraping. This procedure is equivalent to inserting a local orbital bump in a beam transport channel by bending magnets.

Figure 25(a) is the S-POD data obtained with an initially anisotropic plasma under the condition $\nu_{0 x}=\nu_{0 y}$. The transverse rms extent of the plasma was measured with a phosphor screen. The result in the right panel clearly shows a symmetric emittance exchange pattern that suggests the excitation of the expected difference resonance. No ion losses occur during the emittance exchange process as indicated in the left panel. The observed oscillations of the horizontal and vertical plasma sizes are thus caused solely by the emittance exchange. When the LPT operating point is set sufficiently away from the line $\nu_{0 x}-\nu_{0 y}=0$, no emittance exchange takes place as depicted in Fig. 25(b).

Theoretically, many resonances of different orders overlap along the line $k \nu_{0 x}-k \nu_{0 y}=0$. The one driven by the fourth-order $(k=2)$ space-charge potential is known as the Montague resonance [66,67]. The separation of these overlapping difference resonances is a complicated issue. A similar complication is encountered even in noncoupling resonances. For instance, the severe instability observed at $\nu_{0}=1 / 4$ in the GSI UNILAC was interpreted in Ref. [68] as a result from the fourth-order space-charge-driven resonance dominating over the second-order's. It, however, seems uncertain if the effect from the linear resonance, two orders lower than the octupole, is really so weak against the Vlasov prediction about the core stability. In the present paper and other past publications on S-POD experiments, we have consistently taken the simplest standpoint that a lower-order resonance is generally more severe in the beam core than higher-orders'.

As for the resonance overlapping along $k \nu_{0 x}-k \nu_{0 y}=0$, Métral et al. have reported on the evidence of the linear mechanism that leads to a complete emittance exchange between the horizontal and vertical directions [69]. A second-moment analysis in Ref. [57] has also pointed out that the space-charge potential can be a source of linear sum resonance. The signature of such an intensitydependent sum resonance was observed experimentally in the S-POD system [31]. We believe that more investigation 
is necessary to clarify the role of the linear coupling term along the Montague-resonance line. (See the discussion in Appendix C.)

\section{APPENDIX C: SUPPRESSION OF EMITTANCE EXCHANGE ON THIRD-ORDER DIFFERENCE RESONANCES}

We have discovered that the difference resonances with $\left(k, \ell, n^{\prime}\right)=(1,-2,0)$ and $(2,-1,0)$ are no longer active in the initially equipartitioned beam. Other third-order difference resonance bands with $\left(k, \ell, n^{\prime}\right)=(-1,2,1)$ and $(2,-1,1)$ are visible in Fig. 6 . The PIC data in Fig. 8 strongly suggests that the emittance exchange on a particular difference resonance can be suppressed when the initial transverse emittances meet the condition $I_{k \ell}=0$. It is interesting to check if the latter two resonance bands above vanish under this simple condition. Let us look into the case where $(k, \ell)=(2,-1)$. The condition $I_{2,-1}=0$ requires that the horizontal emittance is twice as large as the vertical, i.e., $\varepsilon_{x}=2 \varepsilon_{y}$. The tune diagram in Fig. 26 is based on the WARP simulations performed with this initial emittance ratio. The right and left panels show the results with and without an external nonlinear driving field. As expected, the third-order resonances with $\left(k, \ell, n^{\prime}\right)=$ $(2,-1,0)$ and $(2,-1,1)$ are both eliminated, no matter whether the external driving potential is present. In the case (b), we have introduced the sextupole error $\left(\propto y^{3}-3 x^{2} y\right)$ to enhance the third-order difference resonances with $(k, \ell)=(2,-1)$. While the error field considerably

(a) no error

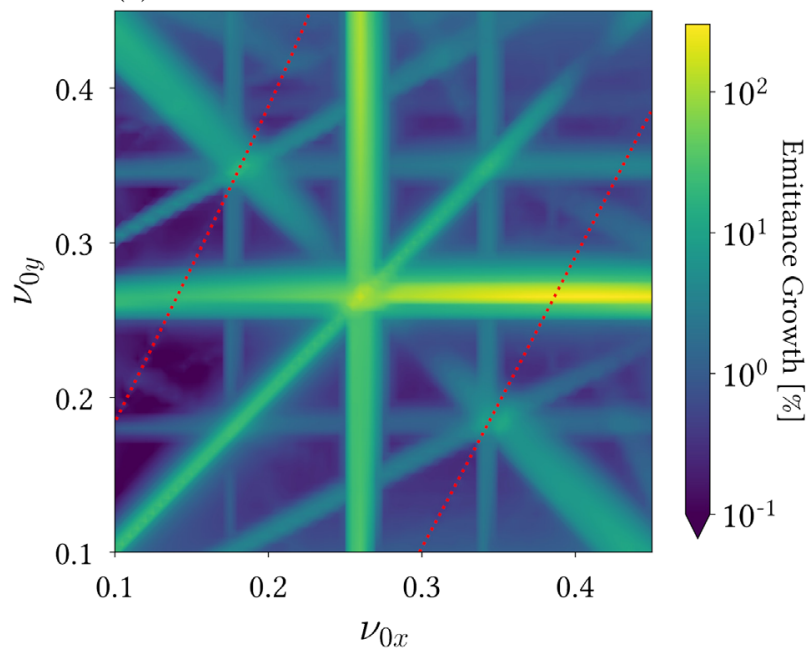

increases the emittance growth along the externalfield-driven resonance lines with $\left(k, \ell, n^{\prime}\right)=(0,3,2)$ and $(2,1,2)$, it has no effect on the difference resonances that satisfy the condition $I_{2,-1}=0$.

This interesting finding could be tested experimentally in existing rings. As shown in Fig. 19, a clear third-order difference resonance band is lying near the regular operating point of the RCS $[63,70]$. Since the sophisticated injection painting scheme is available in this high-power machine, the ratio of the initial transverse emittances can be controlled over a wide range. After accumulating a proton beam with the emittance ratio $\varepsilon_{x} / \varepsilon_{y}=1 / 2$ in a resonancefree area, we move the operating point onto the line $\nu_{0 x}-2 \nu_{0 y}=-6$. If the present understanding is correct, no instability will occur even with the sextupole correction magnets on.

We also recognize another striking disparity between Fig. 26 and other tune diagrams in Sec. III. The resonance bands along $\nu_{0 x}-\nu_{0 y}=0$ has appeared very clearly in Fig. 26 due to the initial emittance imbalance imposed. The coupling instability along this line is usually referred to as the Montague resonance as discussed in Appendix B. It is thought to be driven by the fourth-order space-charge term [66]. The difference and sum resonance lines along $k \nu_{0 x} \pm k \nu_{0 y}=0$ are, however, much clearer than any other nonlinear resonance lines in the absence of the error field. This observation suggests the possibility that the primary source of the difference resonance along $k \nu_{0 x}-k \nu_{0 y}=0$ observed in Fig. 26 is the linear $(k=1)$ skew space-charge

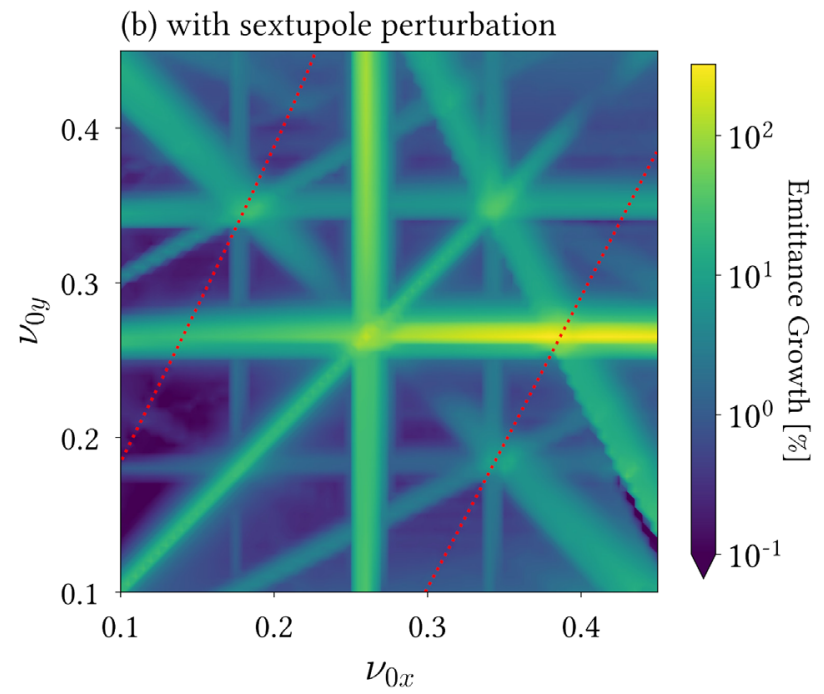

FIG. 26. Tune diagrams obtained from 2D WARP simulations under the initial emittance ratio $\varepsilon_{x} / \varepsilon_{y}=2$. Similarly to Fig. 8, we have used the matched Gaussian beam propagating through the sinusoidal focusing channel of 100 AG periods long. The ratio of the horizontal rms emittance to the vertical is fixed at 2.0 everywhere on the diagram. The beam current is maintained at the same value as assumed in Figs. 4 and 6. The red dotted lines represent the positions of the third-order coherent resonance bands defined by Eq. (8) with $\left(k, \ell, n^{\prime}\right)=(2,-1,0),(2,-1,1)$ and $C_{3}=0.875$, both of which are invisible here. The left panel (a) shows the case where the external force is completely linear. In the right panel (b), the external imperfection potential proportional to $y^{3}-3 x^{2} y$ has been switched on. The perturbation amplitude is set at $1 \%$ of the quadrupole focusing field's. In this case, some particle losses occur near the low-tune boundaries (i.e., in the tail resonance region) of the third-order external-field-driven resonance bands. 
term rather than the fourth-order's $(k=2)$. The existence of such linear parametric resonances driven by the skew potential can be concluded from the 2D Vlasov analysis without the smooth approximation [48].

\section{APPENDIX D: EFFECT OF INITIAL DENSITY DISTORTION}

We here comment on the seed of the self-field-driven parametric instability, specifically, the effect of a finite deviation of an initial particle distribution from the ideal stationary state. Theoretically, nothing serious happens in a perfect matched state even when the operating point is exactly on the parametric resonance. Unlike in real machines, we can easily produce a very well-matched (a) Well-matched

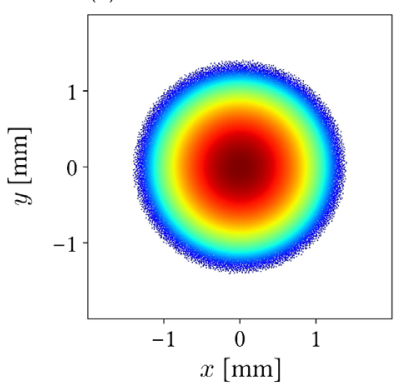

(b) Weakly distorted

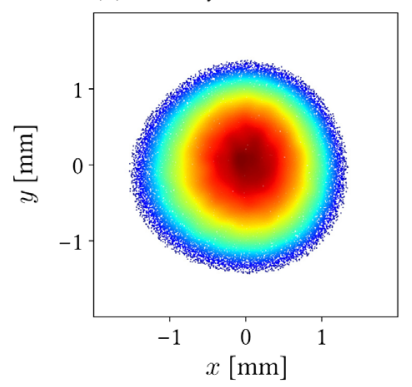

(c) Emittance evolution at $\nu_{0}=0.192$

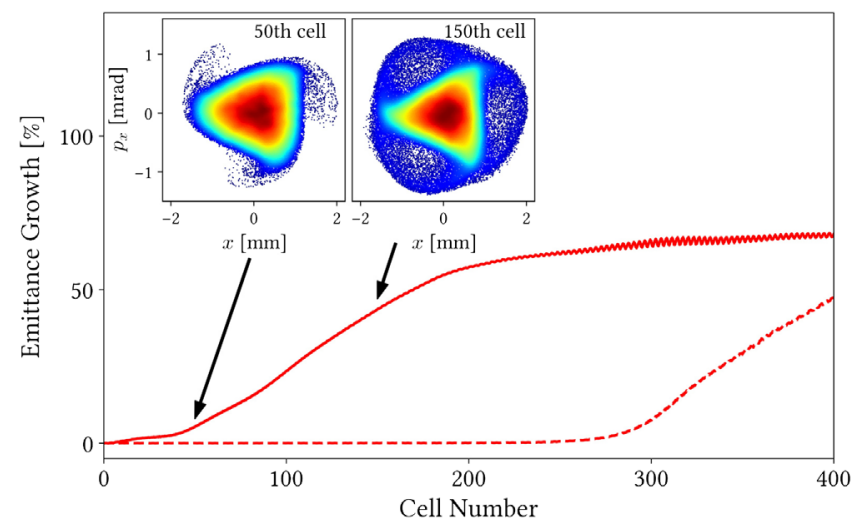

FIG. 27. WARP results on the effect of weak initial distortion to a matched waterbag beam. The tune depression and operating tune are adjusted to the same values as in the case of Fig. 2(a); i.e., $\eta=0.8$ and $\nu_{0}=0.192$. Other numerical conditions are also identical to those assumed in Fig. 2. (a) Initial real-space profile of the waterbag beam well-matched to the lattice. (b) Initial realspace profile of a waterbag beam slightly distorted from the matched state in the left panel. The perturbed distribution was provided through a separate short simulation over a single AG cell with strong normal and skew sextupole potentials. (c) Time evolution of the average emittance growth simulated with the WARP code. The solid line is obtained with the weakly distorted initial distribution while the dashed line with the well-matched distribution. The two insets show the horizontal phase-space configurations of the distorted beam at the 50th cell and at the 150th cell. particle distribution in computer simulations, which almost eliminates the seed of nonlinear coherent resonances. For instance, the lowest-order dipole $(m=1)$ resonance, stronger than the quadrupole instability, usually needs a very long transport distance to get excited in simulations. It is simply because the beam's centroid has been placed precisely on the design orbit at the beginning [39].

A similar argument applies to the excitation of nonlinear modes. Figure 27 is an example demonstrating the effect of weak artificial perturbation to an initial density profile. The upper panels show the cross sections of two waterbag-type beams at the operating point $\nu_{0}=0.192$. The left one is the initial distribution employed for the WARP simulation in Fig. 2(a). A few hundred AG periods were necessary for this well-matched beam to start exhibiting emittance growth due to the coherent sextupole resonance. The distribution in the right panel (b) looks analogous to the left, but we have intentionally introduced a slight distortion of sextupole symmetry. Starting a WARP simulation from this perturbed beam, we see a clear signature of the sextupole core resonance within just a few tens of AG periods as shown in Fig. 27(c).

The tune diagram in Fig. 28 is obtained using the initially distorted waterbag beam. We can identify all possible selffield-driven resonance bands of the second and third orders (though some of them are not very apparent from the picture). As expected, the band widths are narrower than the Gaussian case in Fig. 3. Unlike the Gaussian beam with a long tail, the waterbag beam exhibits a clear core deformation pattern, which makes it a lot easier for us to understand which collective mode is unstable. For

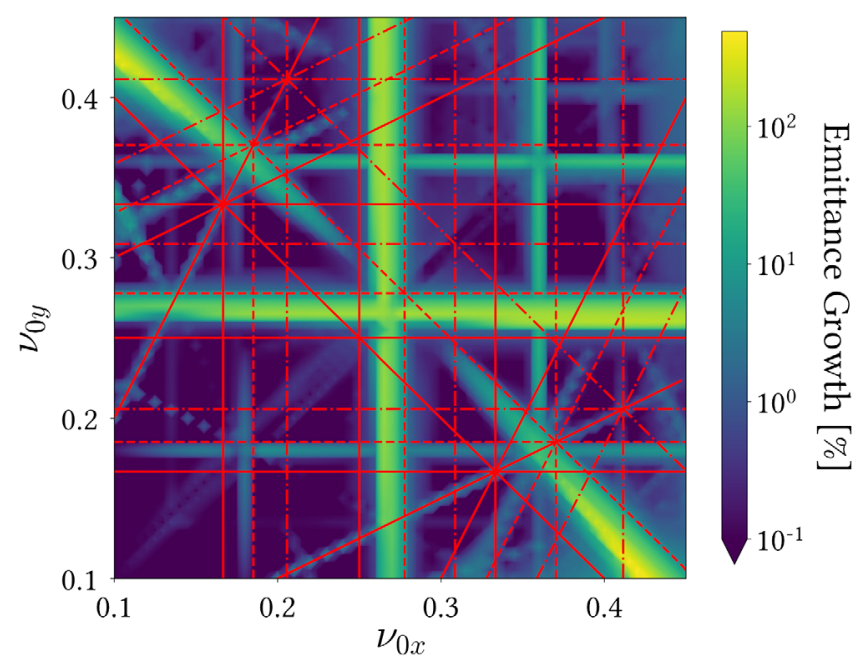

FIG. 28. Tune diagram obtained from 2D WARP simulations with a weakly distorted waterbag distribution. The initial distortion of the sextupole symmetry is developed in the same way as in Fig. 27. The original tune depression before the application of the sextupole distortion is adjusted to $\eta_{x}=\eta_{y}=0.9$ over the whole tune space. Other numerical conditions are identical to those assumed for the Gaussian-based simulations in Fig. 3. 
instance, we always observe the triangular core configuration as in Fig. 2(a) and Fig. 27(c) along the instability bands above $\nu_{0 x(0 y)}=1 / 6$ where the coherent resonance condition in Eq. (8) predicts the instability of the sextupole $(m=3)$ mode.

[1] E. D. Courant and H. S. Snyder, Theory of the alternatinggradient synchrotron, Ann. Phys. (N.Y.) 3, 1 (1958).

[2] Handbook of Accelerator Physics and Engineering, edited by A. W. Chao and M. Tigner (World Scientific, Singapore, 1999) and references therein.

[3] H. Wiedemann, Particle Accelerator Physics (SpringerVerlag, Berlin, 1993).

[4] S. Y. Lee, Accelerator Physics (World Scientific, Singapore, 1999).

[5] M. Conte and W. M. MacKay, An Introduction to the Physics of Particle Accelerators (World Scientific, Singapore, 1991).

[6] N. Madsen, P. Bowe, M. Drewsen, L. H. Hornekær, N. Kjærgaard, A. Labrador, J. S. Nielsen, J. P. Schiffer, P. Shi, and J. S. Hangst, Density Limitation in a Stored LaserCooled Ion Beam, Phys. Rev. Lett. 83, 4301 (1999).

[7] K. Okabe and H. Okamoto, Emittance limitation in cooled hadron beams, Jpn. J. Appl. Phys. 42, 4584 (2003).

[8] It is easy to find many papers using a tune diagram as in Fig. 1. A part of examples presented in recent major accelerator meetings include in the Proceedings of IPAC2018 (JACoW, Geneva, 2018), THPAK05, THPAF054, TUPAL054, THYGBF1; in the Proceedings of HB2018 (JACoW, Geneva, 2018), MOP1WA01, TUA2WD02, WEP2PO024. Detailed information can readily be obtained at the JACoW website, https://www .jacow.org.

[9] I. M. Kapchinskij and V. V. Vladimirskij, Limitations of proton beam current in a strong focusing linear accelerator associated with the beam space charge, in Proceedings of the International Conference on High-Energy Accelerators (CERN, Geneva, 1959), p. 274.

[10] M. Reiser, Theory and Design of Charged Particle Beams (John Wiley \& Sons, New York, 2008) and references therein.

[11] R. L. Gluckstern, Oscillation modes in two-dimensional beams, in Proceedings of the 1970 Linear Accelerator Conference, edited by M. R. Tracy (Fermilab, Batavia, Illinois, 1970), p. 811.

[12] I. Hofmann, L. J. Laslett, L. Smith, and I. Haber, Stability of the Kapchinskij-Vladimirskij (K-V) distribution in long periodic transport systems, Part. Accel. 13, 145 (1983).

[13] H. Okamoto and M. Ikegami, Simulation study of halo formation in breathing round beams, Phys. Rev. E 55, 4694 (1997).

[14] A. V. Fedotov, R. L. Gluckstern, S. S. Kurennoy, and R. D. Ryne, Halo formation in three-dimensional bunches with various phase space distributions, Phys. Rev. ST Accel. Beams 2, 014201 (1999).

[15] T. H. Stix, Waves in Plasmas, (Springer, New York, 1992).

[16] R. C. Davidson, Physics of Nonneutral Plasmas (World Scientific, Singapore, 2001).
[17] A. Piwinski, Intra-beam-scattering, in Proceedings of the 9th International Conference on High Energy Accelerators, Stanford, USA, 1974, pp. 405-409, http://cds.cern.ch/ record/400720.

[18] J. D. Bjorken and S. K. Mtingwa, Intrabeam scattering, Part. Accel. 13, 115 (1983).

[19] L. Smith, Effect of gradient errors in the presence of space charge forces, in Proceedings of the 4th International Conference on High-Energy Accelerators, edited by A. A. Kolomenskij and A. B. Kuznetsov (Dubna, USSR, 1963), p. 1232.

[20] F. J. Sacherer, Ph.D thesis, Lawrence Radiation Laboratory, 1968; Report No. UCRL-18454, 1968.

[21] J. Struckmeier and M. Reiser, Theoretical studies of envelope oscillations and instabilities of mismatched intense charged-particle beams in periodic focusing channels, Part. Accel. 14, 227 (1984).

[22] J. Struckmeier, J. Klabunde, and M. Reiser, On the stability and emittance growth of different particle distributions in a long magnetic quadrupole channel, Part. Accel. 15, 47 (1984).

[23] S. Machida, Space charge effects in low-energy proton synchrotrons, Nucl. Instrum. Methods Phys. Res., Sect. A 309, 43 (1991).

[24] S. M. Lund and R. C. Davidson, Warm-fluid description of intense beam equilibrium and electrostatic stability properties, Phys. Plasmas 5, 3028 (1998).

[25] I. Hofmann, Stability of anisotropic beams with space charge, Phys. Rev. E 57, 4713 (1998).

[26] M. Venturini and R. L. Gluckstern, Resonance analysis for a space charge dominated beam in a circular lattice, Phys. Rev. ST Accel. Beams 3, 034203 (2000).

[27] H. Okamoto and K. Yokoya, Parametric resonances in intense one-dimensional beams propagating through a periodic focusing channel, Nucl. Instrum. Methods Phys. Res., Sect. A 482, 51 (2002).

[28] A. V. Fedotov, I. Hofmann, R. L. Gluckstern, and H. Okamoto, Parametric collective resonances and spacecharge limit in high-intensity rings, Phys. Rev. ST Accel. Beams 6, 094201 (2003).

[29] I. Hofmann, G. Franchetti, O. Boine-Frankenheim, J. Qiang, and R. D. Ryne, Space charge resonances in two and three dimensional anisotropic beams, Phys. Rev. ST Accel. Beams 6, 024202 (2003).

[30] R. Baartman, Betatron resonances with space charge, in Proceedings of Space Charge Physics in High Intensity Hadron Rings (Shelter Island, New York, USA, 1998); AIP Conf. Proc. 448, 56 (1998).

[31] K. Ito, H. Okamoto, Y. Tokashiki, and K. Fukushima, Coherent resonance stop bands in alternating gradient beam transport, Phys. Rev. Accel. Beams 20, 064201 (2017).

[32] S. M. Lund and B. Bukh, Stability properties of the transverse envelope equations describing intense ion beam transport, Phys. Rev. ST Accel. Beams 7, 024801 (2004).

[33] K. Ito, T. Kurauchi, H. Higaki, and H. Okamoto, Experimental observation of low-order collective oscillation modes in a strong focusing lattice, to be published in Proceedings of the 10th International Particle Accelerator 
Conference (IPAC2019), Melbourne, Australia (JACoW, Geneva, 2019), WEPTS018.

[34] K. Ito, M. Matsuba, and H. Okamoto, Effect of quadrupole focusing-field fluctuation on the transverse stability of intense hadron beams in storage rings, Prog. Theor. Exp. Phys. 2018, 023G01 (2018).

[35] K. Fukushima, K. Ito, H. Okamoto, S. Yamaguchi, K. Moriya, H. Higaki, T. Okano, and S. M. Lund, Experimental verification of resonance instability bands in quadrupole doublet focusing channels, Nucl. Instrum. Methods Phys. Res., Sect. A 733, 18 (2014).

[36] S. Ohtsubo, M. Fujioka, H. Higaki, K. Ito, H. Okamoto, H. Sugimoto, and S. M. Lund, Experimental study of coherent betatron resonances with a Paul trap, Phys. Rev. ST Accel. Beams 13, 044201 (2010).

[37] H. Okamoto, K. Fukushima, H. Higaki, D. Ishikawa, K. Ito, T. Iwai, K. Moriya, T. Okano, K. Osaki, and M. Yamaguchi, Beam dynamics studies with non-neutral plasma traps, in Proceedings of the 5th International Particle Accelerator Conference (IPAC2014), Dresden, Germany (JACoW, Geneva, 2014), FRXAA01, p. 4052.

[38] H. Takeuchi, K. Fukushima, K. Ito, K. Moriya, H. Okamoto, and H. Sugimoto, Experimental study of resonance crossing with a Paul trap, Phys. Rev. ST Accel. Beams 15, 074201 (2012).

[39] K. Moriya, M. Ota, K. Fukushima, M. Yamaguchi, K. Ito, and H. Okamoto, Double stop-band structure near halfinteger tunes in high-intensity rings, Phys. Rev. Accel. Beams 19, 114201 (2016).

[40] H. Okamoto, K. Ito, K. Fukushima, and T. Okano, Recent results from the S-POD trap systems on the stability of intense hadron beams, in Proceedings of the 54th ICFA Advanced Beam Dynamics Workshop on High-Intensity, High-Brightness and High-Power Hadron Beams (HB2014), Michigan, USA (JACoW, Geneva, 2015), TUO2LR03, p. 178.

[41] H. Okamoto, M. Endo, K. Fukushima, H. Higaki, K. Ito, K. Moriya, S. Yamaguchi, and S. M. Lund, Experimental simulation of beam propagation over long path lengths using radio-frequency and magnetic traps, Nucl. Instrum. Methods Phys. Res., Sect. A 733, 119 (2014).

[42] H. Okamoto and H. Tanaka, Proposed experiment for the study of beam halo formation, Nucl. Instrum. Methods Phys. Res., Sect. A 437, 178 (1999).

[43] H. Okamoto, Y. Wada, and R. Takai, Radio-frequency quadrupole trap as a tool for experimental beam physics, Nucl. Instrum. Methods Phys. Res., Sect. A 485, 244 (2002).

[44] R. Takai, H. Enokizono, K. Ito, Y. Mizuno, K. Okabe, and H. Okamoto, Development of a compact plasma trap for experimental beam physics, Jpn. J. Appl. Phys. 45, 5332 (2006).

[45] P. K. Ghosh, Ion Traps (Oxford Science, Oxford, 1995).

[46] H. Bartosik, Challenges in understanding space charge effects, ICFA Beam Dynamics Newsletter 75, 26 (2018), and references therein.

[47] I. Hofmann, Space Charge Physics for Particle Accelerators (Springer, New York, 2017) and references therein.

[48] Y. Tokashiki, K. Fukushima, and H. Okamoto, Twodimensional Vlasov analysis of collective resonances in intense charged-particle beams, presented at the 71st Annual meeting of the Physical Society of Japan, 2016 (The Physical Society of Japan, Tokyo, March, 2016), 19aAQ-1 (unpublished).

[49] T. Okano, K. Ito, M. Yamaguchi, K. Moriya, H. Higaki, and H. Okamoto, Experimental study on higher-order resonant instabilities in intense beams with an ion trap, presented at the 71st Annual meeting of the Physical Society of Japan, 2016 (The Physical Society of Japan, Tokyo, March, 2016), 21 pAP-10 (unpublished).

[50] F. J. Sacherer, RMS envelope equations with space charge, IEEE Trans. Nucl. Sci. 18. 1105 (1971).

[51] R. L. Gluckstern, Analytic Model for Halo Formation in High Current Ion Linacs, Phys. Rev. Lett. 73, 1247 (1994).

[52] J. M. Lagniel, On halo formation from space-charge dominated beams, Nucl. Instrum. Methods Phys. Res., Sect. A 345, 46 (1994).

[53] T. P. Wangler, RF Linear Accelerators (John Wiley \& Sons, New York, 1998) and references therein.

[54] D. P. Grote, A. Friedman, G. Craig, I. Haber, and W. Sharp, Progress toward source-to-target simulation, Nucl. Instrum. Methods Phys. Res., Sect. A 464, 563 (2001).

[55] S. M. Lund, T. Kikuchi, and R. C. Davidson, Generation of initial kinetic distributions for simulation of long-pulse charged particle beams with high space-charge intensity, Phys. Rev. ST Accel. Beams 12, 114801 (2009).

[56] J. A. Holmes, V. V. Danilov, J. D. Galambos, D. Jeon, and D. K. Olsen, Space charge dynamics in high intensity rings, Phys. Rev. ST Accel. Beams 2, 114202 (1999).

[57] O. Boine-Frankenheim, I. Hofmann, and J. Struckmeier, Parametric sum envelope instability of periodically focused intense beams, Phys. Plasmas 23, 090705 (2016).

[58] Considering the relatively low beam density $(\eta=0.9)$ and short transport distance (100 AG cells), the very low emittance growth near $\nu_{0 x(0 y)}=1 / 6$ may be due partially to the sixorder effect from the Gaussian tail. See the discussion in Sec. IV.

[59] R. A. Jameson, Beam-intensity limitations in linear accelerators, IEEE Trans. Nucl. Sci. 28, 2408 (1981).

[60] G. Guignard, A general treatment of resonances in accelerators, CERN Report No. CERN 78-11, 1978.

[61] M. Chung, E. P. Gilson, R. C. Davidson, P. C. Efthimion, and R. Majeski, Use of a Linear Paul Trap to Study Random Noise-Induced Beam Degradation in High-Intensity Accelerators, Phys. Rev. Lett. 102, 145003 (2009).

[62] G. Franchetti, I. Hofmann, M. Giovannozzi, M. Martini, and E. Metral, Space charge and octupole driven resonance trapping observed at the CERN Proton Synchrotron, Phys. Rev. ST Accel. Beams 6, 124201 (2003).

[63] H. Hotchi, H. Harada, N. Hayashi, S. Kato, M. Kinsho, K. Okabe, P. K. Saha, Y. Shobuda, F. Tamura, N. Tani, Y. Watanabe, K. Yamamoto, M. Yamamoto, and M. Yoshimoto, Achievement of a low-loss 1-MW beam operation in the 3-GeV rapid cycling synchrotron of the Japan Proton Accelerator Research Complex, Phys. Rev. Accel. Beams 20, 060402 (2017).

[64] H. Hotchi (private communication).

[65] See, e.g., K. Y. Ng, Physics of intensity dependent beam instabilities (World Scientific, Singapore, 2005). 
[66] W. Montague, Fourth-order coupling resonances excited by space-charge forces in a synchrotron, Report No. CERN/ISR/68-38, 1968.

[67] I. Hofmann, G. Franchetti, J. Qiang, and R. Ryne, Dynamical effects in crossing of the Montague resonance, in Proceedings of the 9th European Particle Accelerator Conference (EPAC2004), Lucerne, Switzerland (JACoW, Geneva, 2004), WEPLT053, p. 1960.

[68] L. Groening, W. Barth, W. Bayer, G. Clemente, L. Dahl, P. Forck, P. Gerhard, I. Hofmann, M. S. Kaiser, M. Maier, S. Mickat, T. Milosic, D. Jeon, and D. Uriot, Experimental Evidence of the $90^{\circ}$ Stop Band in the GSI UNILAC, Phys. Rev. Lett. 102, 234801 (2009).
[69] E. Métral, M. Giovannozzi, M. Martini, R. Steerenberg, G. Franchetti, I. Hofmann, J. Qiang, and R. D. Ryne, Space-charge experiments at the CERN Proton Synchrotron, AIP Conf. Proc. 773, 122 (2005).

[70] H. Hotchi, H. Harada, N. Hayashi, M. Kinsho, P. Saha, Y. Shobuda, F. Tamura, K. Yamamoto, Mz. Yamamoto, M. Yoshimoto, S. Kato, Y. Irie, T. Koseki, Y. Sato, K. Satou, and M. Shirakata, Beam commissioning and operation of the Japan Proton Accelerator Research Complex 3-GeV rapid cycling synchrotron, Prog. Theor. Exp. Phys. 2012, 02B003 (2012). 